\title{
Harmonic analysis on the Möbius gyrogroup ${ }^{\dagger}$
}

\author{
Milton Ferreira \\ School of Technology and Management, \\ Polytechnic Institute of Leiria, Portugal \\ 2411-901 Leiria, Portugal. \\ Email: milton.ferreira@ipleiria.pt \\ and
}

CIDMA - Center for Research and Development in Mathematics and Applications,

University of Aveiro, 3810-193 Aveiro, Portugal.

Email mferreira@ua.pt

\begin{abstract}
In this paper we propose to develop harmonic analysis on the Poincaré ball $\mathbb{B}_{t}^{n}$, a model of the $n$-dimensional real hyperbolic space. The Poincaré ball $\mathbb{B}_{t}^{n}$ is the open ball of the Euclidean $n$-space $\mathbb{R}^{n}$ with radius $t>0$, centered at the origin of $\mathbb{R}^{n}$ and equipped with Möbius addition, thus forming a Möbius gyrogroup where Möbius addition in the ball plays the role of vector addition in $\mathbb{R}^{n}$. For any $t>0$ and an arbitrary parameter $\sigma \in \mathbb{R}$ we study the $(\sigma, t)$-translation, the $(\sigma, t)$-convolution, the eigenfunctions of the $(\sigma, t)$-Laplace-Beltrami operator, the $(\sigma, t)$-Helgason Fourier transform, its inverse transform and the associated Plancherel's Theorem, which represent counterparts of standard tools, thus, enabling an effective theory of hyperbolic harmonic analysis. Moreover, when $t \rightarrow+\infty$ the resulting hyperbolic harmonic analysis on $\mathbb{B}_{t}^{n}$ tends to the standard Euclidean harmonic analysis on $\mathbb{R}^{n}$, thus unifying hyperbolic and Euclidean harmonic analysis. As an application we construct diffusive wavelets on $\mathbb{B}_{t}^{n}$.
\end{abstract}

MSC 2000: Primary: 43A85, 42B10 Secondary: 44A35, 20F67

Keywords: Möbius gyrogroup, Helgason-Fourier transform, spherical functions, hyperbolic convolution, eigenfunctions of the Laplace-Beltrami-operator, diffusive wavelets.

\section{Introduction}

Möbius addition, $\oplus$, in the ball $B_{t}^{n}=\left\{x \in \mathbb{R}^{n}:\|x\|<t\right\}$ plays a role analogous to that of vector addition, + , in the Euclidean $n$-space $\mathbb{R}^{n}$, giving rise to the Möbius gyrogroup $\left(B_{t}^{n}, \oplus\right)$, which is analogous to the Euclidean group $\left(\mathbb{R}^{n},+\right)[20,23,25]$. Möbius gyrogroup turn out to be isomorphic, in the gyrovector sense, to corresponding Einstein gyrogroup [23, Sect. 6.19].

The gyrogroup structure is a natural extension of the group structure, discovered in 1988 by A. A. Ungar in the context of Einstein's velocity addition law [18]. The term gyrogroup was coined in 1991 [19] [23, Sect. 1.2], following which it has been extensively studied by A. A. Ungar and others; see,

\footnotetext{
†Accepted author's manuscript (AAM) published in [Journal of Fourier Analysis and Applications 21(2) (2015), 281-317] [DOI: 10.1007/s00041-014-9370-1]. The final publication is available at link.springer.com via http://link . springer.com/article/10.1007\%2Fs00041-014-9370-1
} 
for instance, [21, 10, 9, 16, 23, 24, 26, 27], in the context of abstract algebra, non-Euclidean geometry, mathematical physics, and quantum information and computation.

Möbius addition in the open unit $\operatorname{disc} \mathbb{D}=\{z \in \mathbb{C}:|z|<1\}$ is the well-known binary operation $a \oplus z=(a+z)(1+\bar{a} z)^{-1}, a, z \in \mathbb{D}$, given by a fractional linear transformation. Möbius addition is neither commutative nor associative, but it is both gyrocommutative and gyroassociative under gyrations defined by gyr $[a, b]=(1+a \bar{b}) /(1+\bar{a} b), a, b \in \mathbb{D}$. The generalisation to higher dimensions of the Möbius addition is done by considering Möbius transformations on the ball.

Möbius transformations in $\mathbb{R}^{n}$ were studied by Vahlen in his seminal but almost forgotten paper [29]. Their matricial representation and general properties were rediscovered by Ahlfors [1, 2] almost seventy years later, and independently by Hua [13]. Ahlfors noticed that changing the role of the variables in the Möbius transformation on the ball gives the same Möbius transformation up to a specific orthogonal transformation [1]. This orthogonal transformation (denoted by Ungar as Ahlfors rotation) plays a central role in gyrogroup theory and hyperbolic geometry $[22,23]$ and gives rise to the gyration operator. Moreover, it can be regarded as an analogue of the Thomas precession in the theory of special relativity for the Beltrami-Klein model of hyperbolic geometry governed by Einstein's addition of velocities. By incorporating the gyration operator gyr $[a, b]$ in the algebraic structure, gyrogroup theory repairs the breakdown of associativity and commutativity. In parallel to these advances, Clifford algebras appear as an adequate tool for representation of Möbius transformations (see e.g. [4, 28]). For instance, using the Clifford algebra representation the gyration operator has an explicit spin representation in the case of the Möbius gyrogroup, which in turn allows the construction of explicit factorisations of the ball with respect to Möbius addition [8, 9].

In this paper we propose to study hyperbolic gyroharmonic analysis on the Poincaré ball $\mathbb{B}_{t}^{n}$. With this aim in mind we generalize the results obtained in [17]. The goal of our study is two-fold: first, to understand how the gyration operator affects harmonic analysis on the ball; second, to set the stage for an operator calculus in the framework of wavelet analysis, Gabor analysis, and diffusive wavelets on the ball using the algebraic structure of the Möbius gyrogroup.

In our approach we consider a generalised Laplace-Beltrami operator on the ball depending on the radius $t \in \mathbb{R}^{+}$and on an additional parameter $\sigma \in \mathbb{R}$. This operator is a variation of the common Laplace-Beltrami operator, or conformal Laplacian on the unit ball, which plays an important role in scattering and potential theory. It has connections with other equations like the Weinstein equation (see e.g. [17] and references therein). Using the gyrolanguage we prove new theorems like a Young's inequality for the $(\sigma, t)$-convolution (Theorem 1$)$, the gyrotranslation invariance of the $(\sigma, t)$-convolution (Theorem 2), the gyroassociative law of the $(\sigma, t)$-convolution (Theorem 3 ), and the generalised convolution theorem with respect to the $(\sigma, t)$-Helgason Fourier transform (Theorem 5 ). Each of those theorems involves the gyration operator in a natural way. In the context of the unit ball [17] and symmetric spaces [11, 12] Theorems 3 and 5 are known only in the radial case. In contrast, the gyrogroup theoretic techniques used in this paper enable us to remove the radial condition. It is interesting to explore the translation of these theorems to other models of hyperbolic geometry as, for instance, the upper half space or the hyperboloid, and more generally, Riemannian globally symmetric spaces of noncompact type, but we will not address these problems here.

The paper is organised as follows. In Section 2 we present the Möbius addition in the ball $\mathbb{B}_{t}^{n}$ and its properties. Sections 3 and 4 are dedicated to the study of the $(\sigma, t)$-translation and the $(\sigma, t)$ convolution. In Section 5 we construct the eigenfunctions of the generalised Laplace-Beltrami operator and study the associated $(\sigma, t)$-spherical functions. In Section 6 we define the $(\sigma, t)$-Helgason Fourier transform, which is the relativistic counterpart of the Euclidean Fourier transform. In Section 7 we obtain the inversion formula for the $(\sigma, t)$-Helgason Fourier transform, the Plancherel's Theorem, and 
show that in the limit $t \rightarrow+\infty$ we recover the inverse Fourier transform and Plancherel's Theorem in Euclidean harmonic analysis. Finally, in Section 8 we construct diffusive wavelets on $\mathbb{B}_{t}^{n}$ arising from the heat kernel associated to the generalised Laplace-Beltrami operator $\Delta_{\sigma, t}$. Two appendices, A and $\mathrm{B}$, concerning all necessary facts on spherical harmonics and Jacobi functions, are found at the end of the article.

\section{Möbius addition in the ball}

The Poincaré ball model of $n$-dimensional hyperbolic geometry is the open ball $\mathbb{B}_{t}^{n}=\left\{x \in \mathbb{R}^{n}:\|x\|<\right.$ $t\}$ of $\mathbb{R}^{n}$, endowed with the Poincaré metric

$$
d s^{2}=\frac{d x_{1}^{2}+\ldots+d x_{n}^{2}}{\left(1-\frac{\|x\|^{2}}{t^{2}}\right)^{2}} .
$$

The Poincaré metric is normalised so that in the limit case $t \rightarrow+\infty$ one recovers the Euclidean metric. The group $\mathcal{M}\left(B_{t}^{n}\right)$ of all conformal orientation preserving transformations of $\mathbb{B}_{t}^{n}$ is given by the mappings $K \varphi_{a}$, where $K \in \mathrm{SO}(n)$ and $\varphi_{a}$ are Möbius transformations on $\mathbb{B}_{t}^{n}$ given by (see $[2,9]$ )

$$
\begin{aligned}
\varphi_{a}(x) & =(a+x)\left(1-\frac{a x}{t^{2}}\right)^{-1} \\
& =\frac{\left(1+\frac{2}{t^{2}}\langle a, x\rangle+\frac{1}{t^{2}}\|x\|^{2}\right) a+\left(1-\frac{1}{t^{2}}\|a\|^{2}\right) x}{1+\frac{2}{t^{2}}\langle a, x\rangle+\frac{1}{t^{4}}\|a\|^{2}\|x\|^{2}}
\end{aligned}
$$

where $a, x \in \mathbb{B}_{t}^{n},\langle a, x\rangle$ being the usual scalar product in $\mathbb{R}^{n}$, and $\|x\|$ being the Euclidean norm. Furthermore, $a x$ stands for the Clifford multiplication which we now recall. The Clifford algebra $\mathrm{Cl}_{0, n}$ over $\mathbb{R}^{n}$ is the associative real algebra generated by $\mathbb{R}^{n}$ and $\mathbb{R}$ subject to the relation $x^{2}=-\|x\|^{2}$, for all $x \in \mathbb{R}^{n}$. Therefore, given an orthonormal basis $\left\{e_{j}\right\}_{j=1}^{n}$ of $\mathbb{R}^{n}$ we have the multiplication rules: $e_{j} e_{k}+e_{k} e_{j}=0, j \neq k$, and $e_{j}^{2}=-1, j=1, \ldots, n$. Any non-zero vector $x \in \mathbb{R}^{n}$ is invertible and its inverse is given by $x^{-1}=-\frac{x}{\|x\|^{2}}$. The geometric product between two vectors is given by

$$
x y=\frac{1}{2}(x y+y x)+\frac{1}{2}(x y-y x)
$$

involving the symmetric part $\frac{1}{2}(x y+y x)=-\langle x, y\rangle$ and the anti-symmetric part $\frac{1}{2}(x y-y x):=x \wedge y$, also known as the outer product. The norm in $\mathbb{R}^{n}$ can be extended to $\mathcal{C} l_{0, n}$ and then, for two vectors we have $\|x y\|=\|x\|\|y\|$. This equality is not true for general elements in the Clifford algebra. For more details about the Clifford product and the Clifford norm see [5, 9]. In order to endow the manifold $\mathbb{B}_{t}^{n}$ with an algebraic structure one defines the Möbius addition as

$$
a \oplus x:=\varphi_{a}(x), \quad a, x \in \mathbb{B}_{t}^{n} .
$$

In [9] we proved that $\left(\mathbb{B}_{t}^{n}, \oplus\right)$ is a gyrogroup, i.e., the following properties hold:

(P1) There is a left identity: $0 \oplus a=a$, for all $a \in \mathbb{B}_{t}^{n}$;

(P2) There is a left inverse: $(\ominus a) \oplus a=0$, for all $a \in \mathbb{B}_{t}^{n}$; 
(P3) Möbius addition is gyroassociative, that is, for any $a, b, c \in \mathbb{B}_{t}^{n}$

$$
a \oplus(b \oplus c)=(a \oplus b) \oplus \operatorname{gyr}[a, b] c .
$$

Here gyr $[a, b] c=\frac{1-\frac{a b}{t^{2}}}{\left\|1-\frac{a b}{t^{2}}\right\|} c \frac{1-\frac{b a}{t^{2}}}{\left\|1-\frac{b a}{t^{2}}\right\|}$ is the gyration operator [9], which corresponds to a spin rotation induced by an element of the group $\operatorname{Spin}(n)$ (double covering group of $\mathrm{SO}(n)$ );

(P4) The gyroautomorphism gyr $[a, b]$ possesses the left loop property

$$
\operatorname{gyr}[a, b]=\operatorname{gyr}[a \oplus b, b] .
$$

We remark that $\ominus a=-a$ and Möbius addition (3) corresponds to a left gyrotranslation as defined in [23]. In the limit $t \rightarrow+\infty$, the ball $\mathbb{B}_{t}^{n}$ expands to the whole of the space $\mathbb{R}^{n}$, Möbius addition reduces to vector addition in $\mathbb{R}^{n}$ and, therefore, the gyrogroup $\left(\mathbb{B}_{t}^{n}, \oplus\right)$ reduces to the translation group $\left(\mathbb{R}^{n},+\right)$. The Möbius gyrogroup is gyrocommutative since Möbius addition satisfies the property

$$
a \oplus b=\operatorname{gyr}[a, b](b \oplus a) .
$$

Some useful gyrogroup identities ([23], pp. 48 and 68) that will be used in this article are

$$
\begin{gathered}
\ominus(a \oplus b)=(\ominus a) \oplus(\ominus b) \\
a \oplus((\ominus a) \oplus b)=b \\
(\operatorname{gyr}[a, b])^{-1}=\operatorname{gyr}[b, a] \\
\operatorname{gyr}[a \oplus b, \ominus a]=\operatorname{gyr}[a, b] \\
\operatorname{gyr}[\ominus a, \ominus b]=\operatorname{gyr}[a, b] \\
\operatorname{gyr}[a, \ominus a]=I \\
\operatorname{gyr}[a, b](b \oplus(a \oplus c))=(a \oplus b) \oplus c
\end{gathered}
$$

Properties (8) and (9) are valid for general gyrogroups while properties (6) and (12) are valid only for gyrocommutative gyrogroups. Combining formulas (9) and (12) with (8) we obtain new identities

$$
\begin{gathered}
\operatorname{gyr}[\ominus a, a \oplus b]=\operatorname{gyr}[b, a] \\
b \oplus(a \oplus c)=\operatorname{gyr}[b, a]((a \oplus b) \oplus c) .
\end{gathered}
$$

Möbius transformations (1) satisfy the following useful relations

$$
(a+x)\left(1-\frac{a x}{t^{2}}\right)^{-1}=\left(1-\frac{x a}{t^{2}}\right)^{-1}(a+x)
$$

and

$$
1-\frac{\left\|\varphi_{a}(x)\right\|^{2}}{t^{2}}=\frac{\left(1-\frac{\|a\|^{2}}{t^{2}}\right)\left(1-\frac{\|x\|^{2}}{t^{2}}\right)}{\left\|1-\frac{a x}{t^{2}}\right\|^{2}} .
$$

In the special case when $n=1$, the Möbius gyrogroup becomes a group since gyrations are trivial (a trivial map being the identity map). For $n \geq 2$ the gyrosemidirect product [23] of $\left(\mathbb{B}_{t}^{n}, \oplus\right)$ and $\operatorname{Spin}(n)$ gives a group $\mathbb{B}_{t}^{n} \ltimes_{\text {gyr }} \operatorname{Spin}(n)$ for the operation

$$
\left(a, s_{1}\right)\left(b, s_{2}\right)=\left(a \oplus\left(s_{1} b \overline{s_{1}}\right), \frac{1-\frac{a s_{1} b \overline{s_{1}}}{t^{2}}}{\left\|1-\frac{a s_{1} b \overline{s_{1}}}{t^{2}}\right\|} s_{1} s_{2}\right) .
$$


We remark that this group is a realisation of the proper Lorentz group $\operatorname{Spin}^{+}(1, n)$ (double covering group of $\left.\mathrm{SO}_{0}(1, n)\right)$. In the limit $t \rightarrow+\infty$ the group $\mathbb{B}_{t}^{n} \rtimes_{\text {gyr }} \operatorname{Spin}(n)$ reduces to the Euclidean group $\mathrm{E}(n)=\mathbb{R}^{n} \rtimes \operatorname{Spin}(n)$. The harmonic analysis presented in this paper is associated to the family of Laplace-Beltrami operators $\Delta_{\sigma, t}$ defined by

$$
\Delta_{\sigma, t}=\left(1-\frac{\|x\|^{2}}{t^{2}}\right)\left(\left(1-\frac{\|x\|^{2}}{t^{2}}\right) \Delta-\frac{2 \sigma}{t^{2}}\langle x, \nabla\rangle+\frac{\sigma}{t^{2}}(2-n-\sigma)\right), \quad \sigma \in \mathbb{R}, t \in \mathbb{R}^{+} .
$$

These operators are considered in [17] for the case of the unit ball. The case $\sigma=2-n$ and $t=1$ corresponds to the conformally invariant operator associated to the Poincaré disk model. In the limit $t \rightarrow+\infty$ the operator $\Delta_{\sigma, t}$ reduces to the Laplace operator in $\mathbb{R}^{n}$. Therefore, harmonic analysis associated to $\Delta_{\sigma, t}$ in $\mathbb{B}_{t}^{n}$ provides a link between hyperbolic harmonic analysis and the classic harmonic analysis in $\mathbb{R}^{n}$.

\section{The $(\sigma, t)$-translation}

Definition 1 For a function $f$ defined on $B_{t}^{n}$ and $a \in \mathbb{B}_{t}^{n}$ we define the $(\sigma, t)$-translation $\tau_{a} f$ of $f$ by

$$
\tau_{a} f(x)=f((-a) \oplus x) j_{a}(x)
$$

with

$$
j_{a}(x)=\left(\frac{1-\frac{\|a\|^{2}}{t^{2}}}{\left\|1+\frac{a x}{t^{2}}\right\|^{2}}\right)^{\frac{n+\sigma-2}{2}}=\left(\frac{1-\frac{\|a\|^{2}}{t^{2}}}{1-\frac{2}{t^{2}}\langle a, x\rangle+\frac{\|a\|^{2}\|x\|^{2}}{t^{4}}}\right)^{\frac{n+\sigma-2}{2}} .
$$

The multiplicative factor $j_{a}(x)$ is a positive function and agrees with the Jacobian of the transformation $\varphi_{-a}(x)=(-a) \oplus x$ when $\sigma=n+2$. In the case $\sigma=2-n$ the $(\sigma, t)$-translation reduces to $\tau_{a} f(x)=$ $f((-a) \oplus x)$. Moreover, for any $\sigma \in \mathbb{R}$, we obtain in the limit $t \rightarrow+\infty$ the Euclidean translation operator $\tau_{a} f(x)=f(-a+x)=f(x-a)$.

Lemma 1 For any $a, b, x, y \in \mathbb{B}_{t}^{n}$ the following relations hold

$$
\begin{aligned}
\text { (i) } & j_{-a}(-x)=j_{a}(x) \\
(\text { ii }) & j_{a}(a) j_{a}(0)=1 \\
(\text { iii }) & j_{a}(x)=j_{x}(a) j_{a}(0) j_{x}(x) \\
(\text { iv }) & j_{a}(a \oplus x)=\left(j_{-a}(x)\right)^{-1} \\
(v) & j_{(-a) \oplus x}(0)=j_{x \oplus(-a)}(0)=j_{x}(a) j_{a}(0)=j_{a}(x) j_{x}(0) \\
(\text { vi }) & j_{(-a) \oplus x}((-a) \oplus x)=\left(j_{a}(x)\right)^{-1} j_{x}(x) \\
(v i i) & \tau_{a} j_{y}(x)=\left[\tau_{-a} j_{x}(y)\right] j_{x}(x) j_{y}(0) \\
(v i i i) & \tau_{-a} j_{a}(x)=1 \\
(i x) & \tau_{a} j_{y}(x)=j_{a \oplus y}(x) \\
(x) & \tau_{a} f(x)=\left[\tau_{x} f(-\operatorname{gyr}[x, a] a)\right] j_{a}(0) j_{x}(x) \\
(x i) & \tau_{b} \tau_{a} f(x)=\tau_{b \oplus a} f(\operatorname{gyr}[a, b] x) \\
(x i i) & \tau_{-a} \tau_{a} f(x)=f(x) \\
(x i i i) & \tau_{b} \tau_{a} f(x)=\left[\tau_{-b} \tau_{x} f(-\operatorname{gyr}[-b, x \oplus a] \operatorname{gyr}[x, a] a)\right] j_{a}(0) j_{x}(x) .
\end{aligned}
$$


Proof: In the proof we use the following properties of the Clifford product:

$$
a^{2}=-\|a\|^{2} \quad \text { and } \quad\|a b\|=\|a\|\|b\| \quad \text { for any } a, b \in \mathbb{B}_{t}^{n} \subset \mathbb{R}^{n} .
$$

Identities (19)-(21) can be easily verified by definition. Now we prove equality (22):

$$
\begin{aligned}
j_{a}(a \oplus x) & =\left(\frac{1-\frac{\|a\|^{2}}{t^{2}}}{\left\|1+\frac{a}{t^{2}}(a+x)\left(1-\frac{a x}{t^{2}}\right)^{-1}\right\|^{2}}\right)^{\frac{n+\sigma-2}{2}} \\
& =\left(\frac{1-\frac{\|a\|^{2}}{t^{2}}}{\left\|1-\frac{a x}{t^{2}}+\frac{a}{t^{2}}(a+x)\right\|^{2}\left\|1-\frac{a x}{t^{2}}\right\|^{-2}}\right)^{\frac{n+\sigma-2}{2}} \\
& =\left(\frac{\left\|1-\frac{a x}{t^{2}}\right\|^{2}}{1-\frac{\|a\|^{2}}{t^{2}}}\right)^{\frac{n+\sigma-2}{2}} \\
& =\left(j_{-a}(x)\right)^{-1} .
\end{aligned}
$$

Equality (23) follows from (16):

$$
\begin{aligned}
j_{(-a) \oplus x}(0)=\left(1-\frac{\left\|\varphi_{-a}(x)\right\|^{2}}{t^{2}}\right)^{\frac{n+\sigma-2}{2}} & =\left(\frac{\left(1-\frac{\|a\|^{2}}{t^{2}}\right)\left(1-\frac{\|x\|^{2}}{t^{2}}\right)}{\left\|1+\frac{a x}{t^{2}}\right\|^{2}}\right)^{\frac{n+\sigma-2}{2}} \\
& =j_{a}(x) j_{x}(0) \\
& =j_{x}(a) j_{a}(0) .
\end{aligned}
$$

Equality (24) follows from (20) and (23) since we have

$$
j_{(-a) \oplus x}((-a) \oplus x)=\left(j_{(-a) \oplus x}(0)\right)^{-1}=\left(j_{a}(x) j_{x}(0)\right)^{-1}=\left(j_{a}(x)\right)^{-1} j_{x}(x) .
$$

To prove equality (25) we note first that by (16) we can write $j_{a}(x)$ as

$$
j_{a}(x)=\left(\frac{1-\frac{\left\|\varphi_{-a}(x)\right\|^{2}}{t^{2}}}{1-\frac{\|x\|^{2}}{t^{2}}}\right)^{\frac{n+\sigma-2}{2}} .
$$

By definition we have

$$
\tau_{a} j_{y}(x)=j_{y}((-a) \oplus x) j_{a}(x)=j_{y}\left(\varphi_{-a}(x)\right) j_{a}(x)
$$

Since

$$
\begin{array}{rlr}
\varphi_{-y}\left(\varphi_{-a}(x)\right) & =(-y) \oplus((-a) \oplus x) \quad \text { (by }(3)) \\
& =-(y \oplus(a \oplus(-x))) \quad(\text { by }(6)) \\
& =-\operatorname{gyr}[y, a]((a \oplus y) \oplus(-x)) \quad(\text { by }(14)) \\
& =-\operatorname{gyr}[y, a] \varphi_{a \oplus y}(-x) \quad(\text { by }(3))
\end{array}
$$


then $\left\|\varphi_{-y}\left(\varphi_{-a}(x)\right)\right\|=\left\|\varphi_{a \oplus y}(-x)\right\|$ and by (32) and (16) we obtain

$$
\begin{aligned}
j_{y}\left(\varphi_{-a}(x)\right) & =\left(\frac{1-\frac{\left\|\varphi_{a \oplus y}(-x)\right\|^{2}}{t^{2}}}{1-\frac{\left\|\varphi_{-a}(x)\right\|^{2}}{t^{2}}}\right)^{\frac{n+\sigma-2}{2}} \\
& =\left(\frac{\left(1-\frac{\|a \oplus y\|^{2}}{t^{2}}\right)\left(1-\frac{\|x\|^{2}}{t^{2}}\right)}{\left\|1+\frac{x a \oplus y}{t^{2}}\right\|^{2}\left(1-\frac{\|\varphi-a(x)\|^{2}}{t^{2}}\right)}\right)^{\frac{n+\sigma-2}{2}} \\
& =\left(\frac{\left(1-\frac{\|a\|^{2}}{t^{2}}\right)\left(1-\frac{\|y\|^{2}}{t^{2}}\right)\left(1-\frac{\|x\|^{2}}{t^{2}}\right)}{\left\|1-\frac{a y}{t^{2}}\right\|^{2}\left\|1+\frac{x a \oplus y}{t^{2}}\right\|^{2}\left(1-\frac{\left\|\varphi_{-a}(x)\right\|^{2}}{t^{2}}\right)}\right)^{\frac{n+\sigma-2}{2}} .
\end{aligned}
$$

Therefore, by (34) and (26) we obtain

$$
\begin{aligned}
\tau_{a} j_{y}(x) & =j_{y}\left(\varphi_{-a}(x)\right) j_{a}(x) \\
& =j_{x}(a \oplus y) j_{-a}(y) j_{x}(x) j_{y}(0) \\
& =\left[\tau_{-a} j_{x}(y)\right] j_{x}(x) j_{y}(0) .
\end{aligned}
$$

The proof of this identity can also be done using definition (18) with the Clifford product. Equality (26) follows from (22):

$$
\tau_{-a} j_{a}(x)=j_{a}(a \oplus x) j_{-a}(x)=\left(j_{-a}(x)\right)^{-1} j_{-a}(x)=1 .
$$

Equality (27) follows from (33) and (32):

$$
\tau_{a} j_{y}(x)=j_{y}\left(\varphi_{-a}(x)\right) j_{a}(x)=j_{a \oplus y}(x) .
$$

To prove (28) we have the following identities:

$$
\begin{aligned}
{\left[\tau_{x} f(-\operatorname{gyr}[x, a] a)\right] j_{a}(0) j_{x}(x) } & =f(-\operatorname{gyr}[x,(-x) \oplus a]((-x) \oplus a)) j_{x}(a) j_{a}(0) j_{x}(x) \\
& =f(-\operatorname{gyr}[a,-x]((-x) \oplus a)) j_{a}(x) \quad(\text { by }(13),(21)) \\
& =f(-(a \oplus(-x))) j_{a}(x) \quad(\text { by }(5)) \\
& =f((-a) \oplus x) j_{a}(x) \quad(\text { by }(6)) \\
& =\tau_{a} f(x) .
\end{aligned}
$$

Now we prove equality (29):

$$
\begin{aligned}
\tau_{b} \tau_{a} f(x) & =f((-a) \oplus((-b) \oplus x)) \tau_{b} j_{a}(x) \quad(\text { by }(17)) \\
& \left.=f(\operatorname{gyr}[-a,-b](((-b) \oplus(-a)) \oplus x)) j_{b \oplus a}(x) \quad \text { (by }(14),(27)\right) \\
& =f(\operatorname{gyr}[a, b]((-(b \oplus a)) \oplus x)) j_{b \oplus a}(x) \quad(\text { by }(10),(6)) \\
& =\tau_{b \oplus a} f(\operatorname{gyr}[a, b] x) \quad(\text { by }(17)) .
\end{aligned}
$$

Equality (30) follows from (29) considering $b=-a$ since $(-a) \oplus a=0$ and gyr $[a,-a]=I$ by (11). Finally, we prove the last identity: 


$$
\begin{aligned}
{\left[\tau_{-b} \tau_{x}\right.} & f(-\operatorname{gyr}[-b, x \oplus a] \operatorname{gyr}[x, a] a)] j_{a}(0) j_{x}(x)= \\
= & {\left.\left[\tau_{-b} f(-\operatorname{gyr}[-b, x \oplus((-x) \oplus a))] \operatorname{gyr}[x,(-x) \oplus a]((-x) \oplus a)\right) j_{x}(a)\right] } \\
& \times j_{a}(0) j_{x}(x) \quad(\operatorname{by}(17)) \\
= & {[f(-\operatorname{gyr}[-b, b \oplus a] \operatorname{gyr}[x,(-x) \oplus(b \oplus a)]((-x) \oplus(b \oplus a))) \times} \\
& \left.\times\left(\tau_{-b} j_{x}(a)\right)\right] j_{a}(0) j_{x}(x) \quad(\operatorname{by}(17),(7)) \\
= & f(-\operatorname{gyr}[a, b] \operatorname{gyr}[b \oplus a,-x]((-x) \oplus(b \oplus a))) \tau_{b} j_{a}(x) \quad(\text { by } \quad(13),(25)) \\
= & f(-\operatorname{gyr}[a, b]((b \oplus a) \oplus(-x))) \tau_{b} j_{a}(x) \quad(\text { by }(5)) \\
= & f(-(a \oplus(b \oplus(-x)))) \tau_{b} j_{a}(x) \quad(\text { by }(14)) \\
= & f((-a) \oplus((-b) \oplus x)) \tau_{b} j_{a}(x) \quad(\text { by }(6)) \\
= & \tau_{b} f((-a) \oplus x) j_{a}(x) \quad(\text { by } \quad(17)) \\
= & \tau_{b} \tau_{a} f(x) \quad(\text { by }(17)) .
\end{aligned}
$$

Corollary 1 Let $f$ be a radial function defined on $\mathbb{B}_{t}^{n}$, i.e. $f(x)=f(\|x\|), \forall x \in \mathbb{B}_{t}^{n}$. Then for any $a, b, x \in \mathbb{B}_{t}^{n}$ we have

$$
\begin{aligned}
& \text { (i) } \tau_{a} f(x)=\left[\tau_{x} f(a)\right] j_{a}(0) j_{x}(x) ; \\
& \text { (ii) } \tau_{b} \tau_{a} f(x)=\tau_{b \oplus a} f(x) \text {; } \\
& \text { (iii) } \tau_{b} \tau_{a} f(x)=\left[\tau_{-b} \tau_{x} f(a)\right] j_{a}(0) j_{x}(x) .
\end{aligned}
$$

Before we prove that the generalised Laplace-Beltrami operator $\Delta_{\sigma, t}$ commutes with $(\sigma, t)$-translations we present a representation formula for the operator $\Delta_{\sigma, t}$ using the Laplace operator in $\mathbb{R}^{n}$.

Proposition 1 For each $f \in C^{2}\left(\mathbb{B}_{t}^{n}\right)$ and $a \in \mathbb{B}_{t}^{n}$

$$
\left(\Delta_{\sigma, t} f\right)(a)=\left(j_{a}(0)\right)^{-1} \Delta\left(\tau_{-a} f\right)(0)+\frac{\sigma(2-n-\sigma)}{t^{2}} f(a)
$$

Proof: Let $a \in \mathbb{B}_{t}^{n}$ and denote by $T_{1}, \ldots, T_{n}$ the coordinates of the mapping $\varphi_{a}(x)$. Then by the chain rule we have

$$
\begin{aligned}
\Delta\left(\tau_{-a} f\right)(0)= & {\left[\sum_{j, k=1}^{n} \frac{\partial^{2} f}{\partial x_{j} \partial x_{k}}(a) \sum_{i=1}^{n} \frac{\partial T_{k}}{\partial x_{i}}(0) \frac{\partial T_{j}}{\partial x_{i}}(0)+\sum_{k=1}^{n} \frac{\partial f}{\partial x_{k}}(a) \sum_{i=1}^{n} \frac{\partial^{2} T_{k}}{\partial x_{i}^{2}}(0)\right] j_{-a}(0)+} \\
& +2 \sum_{k=1}^{n} \frac{\partial f}{\partial x_{k}}(a) \sum_{i=1}^{n} \frac{\partial T_{k}}{\partial x_{i}}(0) \frac{\partial j_{-a}}{\partial x_{i}}(0)+f(a) \sum_{i=1}^{n} \frac{\partial^{2} j_{-a}}{\partial x_{i}^{2}}(0) .
\end{aligned}
$$

Since

$$
\frac{\partial T_{k}}{\partial x_{i}}(0)=\left(1-\frac{\|a\|^{2}}{t^{2}}\right) \delta_{k, i} \quad \frac{\partial^{2} T_{k}}{\partial x_{i}^{2}}(0)=\left(1-\frac{\|a\|^{2}}{t^{2}}\right) \frac{\left(2 a_{k}-4 a_{i} \delta_{k, i}\right)}{t^{2}}
$$

and

$$
\frac{\partial j_{-a}}{\partial x_{i}}(0)=-j_{a}(0)(n+\sigma-2) \frac{a_{i}}{t^{2}} \frac{(2-n-\sigma)}{2}
$$




$$
\frac{\partial^{2} j_{-a}}{\partial x_{i}^{2}}(0)=j_{a}(0) \frac{(n+\sigma-2)\left((n+\sigma) a_{i}^{2}-\|a\|^{2}\right)}{t^{2}} .
$$

we obtain by putting $\mu_{a}=1-\frac{\|a\|^{2}}{t^{2}}$

$$
\begin{aligned}
\Delta\left(\tau_{-a} f\right)(0)= & j_{a}(0) \mu_{a}\left(\mu_{a} \Delta f(a)-\frac{2 \sigma}{t^{2}} \sum_{k=1}^{n} \frac{\partial f}{\partial x_{k}}(a) a_{k}+\frac{\sigma(2-n-\sigma)}{t^{2}} f(a)\right) \\
& -\frac{\sigma(2-n-\sigma)}{t^{2}} f(a) j_{a}(0) \\
= & j_{a}(0)\left(\left(\Delta_{\sigma, t} f\right)(a)-\frac{\sigma(2-n-\sigma)}{t^{2}} f(a)\right) .
\end{aligned}
$$

Therefore, we get

$$
\left(\Delta_{\sigma, t} f\right)(a)=\left(j_{a}(0)\right)^{-1} \Delta\left(\tau_{-a} f\right)(0)+\frac{\sigma(2-n-\sigma)}{t^{2}} f(a) .
$$

Proposition 2 The operator $\Delta_{\sigma, t}$ commutes with $(\sigma, t)$-translations, i.e.

$$
\Delta_{\sigma, t}\left(\tau_{b} f\right)=\tau_{b}\left(\Delta_{\sigma, t} f\right) \quad \forall f \in C^{2}\left(\mathbb{B}_{t}^{n}\right), \forall b \in \mathbb{B}_{t}^{n} .
$$

Proof: Using (38) we have

$$
\begin{aligned}
\Delta_{\sigma, t}\left(\tau_{b} f\right)(a)= & \left(j_{a}(0)\right)^{-1} \Delta\left(\tau_{-a} \tau_{b} f\right)(0)+\frac{\sigma(2-n-\sigma)}{t^{2}} \tau_{b} f(a) \\
= & \left.\left(j_{a}(0)\right)^{-1} \Delta\left(f((-b) \oplus(a \oplus x)) \tau_{-a} j_{b}(x)\right)\right|_{x=0}+ \\
& +\frac{\sigma(2-n-\sigma)}{t^{2}} f((-b) \oplus a) j_{b}(a) .
\end{aligned}
$$

Now, since

$$
(-b) \oplus(a \oplus x)=((-b) \oplus a) \oplus \operatorname{gyr}[-b, a] x \quad(\text { by }(4))
$$

and

$$
\begin{array}{rlrl}
\tau_{-a} j_{b}(x) & =j_{(-a) \oplus b}(x) \quad(\text { by }(27)) & \\
& =j_{b \oplus(-a)}(\operatorname{gyr}[b,-a] x) & & (\text { by }(5),(8)) \\
& =j_{b \oplus(-a)}(\operatorname{gyr}[-b, a] x) & & (\text { by }(10))
\end{array}
$$

then together with the invariance of $\Delta$ under the group $\mathrm{SO}(n),(23)$ and (19) we obtain

$$
\begin{aligned}
\Delta_{\sigma, t}\left(\tau_{b} f\right)(a)= & \left.\left(j_{a}(0)\right)^{-1} \Delta\left(f(((-b) \oplus a) \oplus \operatorname{gyr}[-b, a] x) j_{b \oplus(-a)}(\operatorname{gyr}[-b, a] x)\right)\right|_{x=0} \\
& +\frac{\sigma(2-n-\sigma)}{t^{2}} f((-b) \oplus a) j_{b}(a) \\
= & \left(j_{a}(0)\right)^{-1} \Delta\left(\tau_{-((-b) \oplus a)} f\right)(0)+\frac{\sigma(2-n-\sigma)}{t^{2}} f((-b) \oplus a) j_{b}(a) \\
= & \left(j_{(-b) \oplus a}(0)\right)^{-1} j_{b}(a) \Delta\left(\tau_{-((-b) \oplus a)} f\right)(0)+\frac{\sigma(2-n-\sigma)}{t^{2}} f((-b) \oplus a) j_{b}(a) \\
= & \left(\Delta_{\sigma, t} f\right)((-b) \oplus a) j_{b}(a) \\
= & \tau_{b}\left(\Delta_{\sigma, t} f\right)(a) .
\end{aligned}
$$


For studying some $L^{2}$-properties of the invariant Laplace $\Delta_{\sigma, t}$ and the $(\sigma, t)$-translation we consider the weighted Hilbert space $L^{2}\left(\mathbb{B}_{t}^{n}, d \mu_{\sigma, t}\right)$ with

$$
d \mu_{\sigma, t}(x)=\left(1-\frac{\|x\|^{2}}{t^{2}}\right)^{\sigma-2} d x,
$$

where $d x$ stands for the Lebesgue measure in $\mathbb{R}^{n}$. For the special case $\sigma=2-n$ we recover the invariant measure associated to the Möbius transformations $\varphi_{a}(x)$.

Proposition 3 For $f, g \in L^{2}\left(\mathbb{B}_{t}^{n}, d \mu_{\sigma, t}\right)$ and $a \in \mathbb{B}_{t}^{n}$ we have

$$
\int_{\mathbb{B}_{t}^{n}} \tau_{a} f(x) \overline{g(x)} d \mu_{\sigma, t}(x)=\int_{\mathbb{B}_{t}^{n}} f(x) \overline{\tau_{-a} g(x)} d \mu_{\sigma, t}(x) .
$$

Proof: By definition we have

$$
I=\int_{\mathbb{B}_{t}^{n}} \tau_{a} f(x) \overline{g(x)} d \mu_{\sigma, t}(x)=\int_{\mathbb{B}_{t}^{n}} f((-a) \oplus x) j_{a}(x) \overline{g(x)} d \mu_{\sigma, t}(x) .
$$

Making the change of variables $(-a) \oplus x=z$, which is equivalent by (7) to $x=a \oplus z$, the measure becomes

$$
\begin{aligned}
d \mu_{\sigma, t}(a \oplus z) & =\left(\frac{1-\frac{\|a\|^{2}}{t^{2}}}{\left\|1-\frac{a z}{t^{2}}\right\|^{2}}\right)^{n}\left(1-\frac{\|a \oplus z\|^{2}}{t^{2}}\right)^{\sigma-2} d z \\
& \left.=\left(j_{-a}(z)\right)^{2} d \mu_{\sigma, t}(z) \text { (by }(16),(18)\right) .
\end{aligned}
$$

Therefore, it follows

$$
\begin{aligned}
I & =\int_{\mathbb{B}_{t}^{n}} f(z) j_{a}(a \oplus z) \overline{g(a \oplus z)}\left(j_{-a}(z)\right)^{2} d \mu_{\sigma, t}(z) \\
& =\int_{\mathbb{B}_{t}^{n}} f(z) \overline{g(a \oplus z)} \tau_{-a} j_{a}(z) j_{-a}(z) d \mu_{\sigma, t}(z) \\
& =\int_{\mathbb{B}_{t}^{n}} f(z) \overline{\tau_{-a} g(z)} d \mu_{\sigma, t}(z) \quad(\text { by }(26)) .
\end{aligned}
$$

Corollary 2 For $f, g \in L^{2}\left(\mathbb{B}_{t}^{n}, d \mu_{\sigma, t}\right)$ and $a \in \mathbb{B}_{t}^{n}$ we have

$$
\begin{aligned}
& \text { (i) } \int_{\mathbb{B}_{t}^{n}} \tau_{a} f(x) d \mu_{\sigma, t}(x)=\int_{\mathbb{B}_{t}^{n}} f(x) j_{-a}(x) d \mu_{\sigma, t}(x) ; \\
& \text { (ii) If } \sigma=2-n \text { then } \int_{\mathbb{B}_{t}^{n}} \tau_{a} f(x) d \mu_{\sigma, t}(x)=\int_{\mathbb{B}_{t}^{n}} f(x) d \mu_{\sigma, t}(x) ; \\
& \text { (iii) }\left\|\tau_{a} f\right\|_{2}=\|f\|_{2} .
\end{aligned}
$$

From Corollary 2 we see that the $(\sigma, t)$-translation $\tau_{a}$ is an unitary operator in $L^{2}\left(\mathbb{B}_{t}^{n}, d \mu_{\sigma, t}\right)$ and the measure $d \mu_{\sigma, t}$ is translation invariant only for the case $\sigma=2-n$.

An important property of the Laplace operator in $\mathbb{R}^{n}$ is that it is a self-adjoint operator. The same holds for the hyperbolic operator $\Delta_{\sigma, t}$ due to the representation formula (38) (see [17] for the proof in the case $t=1)$. 


\section{The $(\sigma, t)$-convolution}

In this section we define the $(\sigma, t)$-convolution of two functions, we study its properties and we establish the respective Young's inequality and gyroassociative law. In the limit $t \rightarrow+\infty$ both definitions and properties tend to their Euclidean counterparts.

Definition 2 The $(\sigma, t)$-convolution of two measurable functions $f$ and $g$ is given by

$$
(f * g)(x)=\int_{\mathbb{B}_{t}^{n}} f(y) \tau_{x} g(-y) j_{x}(x) d \mu_{\sigma, t}(y), \quad x \in \mathbb{B}_{t}^{n} .
$$

The $(\sigma, t)$-convolution is commutative, i.e., $f * g=g * f$. This can be seen by (39) and a change of variables $z \mapsto-y$. It is well defined only for $\sigma<1$ as the next proposition shows.

Proposition 4 For $\sigma<1$ and $f, g \in L^{1}\left(\mathbb{B}_{t}^{n}, d \mu_{\sigma, t}\right)$ the $(\sigma, t)$-convolution is well defined and satisfies the inequality

$$
\|f * g\|_{1} \leq C_{\sigma}\|f\|_{1}\|\widetilde{g}\|_{1}
$$

where $\widetilde{g}(r)=\operatorname{ess}_{\substack{\xi \in \mathbb{S}^{n-1} \\ y \in \mathbb{B}_{t}^{n}}} g(\operatorname{gyr}[y, r \xi] r \xi)$ for any $r \in[0, t[$, and

$$
C_{\sigma}=\left\{\begin{array}{cl}
1, & \text { if } \sigma \in] 2-n, 0[ \\
\frac{\Gamma(n / 2) \Gamma(1-\sigma)}{\Gamma\left(\frac{2-\sigma}{2}\right) \Gamma\left(\frac{n-\sigma}{2}\right)}, & \text { if } \sigma \in]-\infty, 2-n] \cup[0,1[
\end{array} .\right.
$$

Proof: Using (28), (20), (41), and (19) we have

$$
\begin{aligned}
I & =\int_{\mathbb{B}_{t}^{n}}\left|\tau_{x} g(-y)\right| j_{x}(x) d \mu_{\sigma, t}(x) \\
& =\int_{\mathbb{B}_{t}^{n}}\left|\tau_{y} g(\operatorname{gyr}[y, x] x)\right| j_{x}(0) j_{y}(y) j_{x}(x) d \mu_{\sigma, t}(x) \\
& =\int_{\mathbb{B}_{t}^{n}}|g(\operatorname{gyr}[y, x] x)| j_{-y}(x) j_{-y}(-y) d \mu_{\sigma, t}(x) \\
& =A_{n-1} \int_{0}^{t}\left(1-\frac{r^{2}}{t^{2}}\right)^{\sigma-2} r^{n-1} d r \int_{\mathbb{S}^{n-1}}|g(\operatorname{gyr}[y, r \xi] r \xi)| j_{-y}(r \xi) j_{-y}(-y) d \sigma(\xi) .
\end{aligned}
$$

In the last equality we have used polar coordinates $r \xi$, with $r \in\left[0, t\left[\right.\right.$ and $\xi \in \mathbb{S}^{n-1}$, and the normalised surface area $d \sigma(\xi)=d \xi / A_{n-1}$, with $A_{n-1}$ being the surface area of $\mathbb{S}^{n-1}$. For each $r \in[0, t[$ we

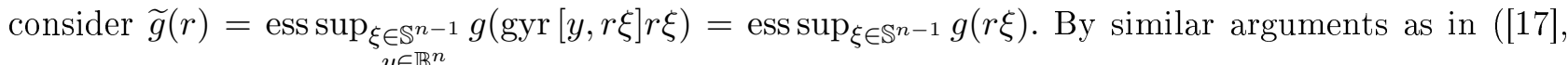
Lemma 3.12) we have

$$
\int_{\mathbb{S}^{n-1}} j_{-y}(r \xi) j_{-y}(-y) d \sigma(\xi) \leq C_{\sigma}
$$

for all $y \in \mathbb{B}_{t}^{n}, r \in[0, t[$, and $\sigma<1$. Therefore,

$$
\int_{\mathbb{B}_{t}^{n}}\left|\tau_{x} g(-y)\right| j_{x}(x) d \mu_{\sigma, t}(x) \leq C_{\sigma}\|\widetilde{g}\|_{1} .
$$


Finally,

$$
\begin{aligned}
\|f * g\|_{1} & =\int_{\mathbb{B}_{t}^{n}}\left|\int_{\mathbb{B}_{t}^{n}} f(y) \tau_{x} g(-y) j_{x}(x) d \mu_{\sigma, t}(y)\right| d \mu_{\sigma, t}(x) \\
& \leq \int_{\mathbb{B}_{t}^{n}} \int_{\mathbb{B}_{t}^{n}}|f(y)|\left|\tau_{x} g(-y)\right| j_{x}(x) d \mu_{\sigma, t}(y) d \mu_{\sigma, t}(x) \\
& =\int_{\mathbb{B}_{t}^{n}}|f(y)|\left(\int_{\mathbb{B}_{t}^{n}}\left|\tau_{x} g(-y)\right| j_{x}(x) d \mu_{\sigma, t}(x)\right) d \mu_{\sigma, t}(y) \\
& \leq C_{\sigma}\|f\|_{1}\|\widetilde{g}\|_{1} .
\end{aligned}
$$

In the special case when $g$ is a radial function we obtain as a corollary that $\|f * g\|_{1} \leq C_{\sigma}\|f\|_{1}\|g\|_{1}$ since $\widetilde{g}=g$. We can also prove that for $f \in L^{\infty}\left(\mathbb{B}_{t}^{n}, d \mu_{\sigma, t}\right)$ and $g \in L^{1}\left(\mathbb{B}_{t}^{n}, d \mu_{\sigma, t}\right)$ we have the inequality

$$
\|f * g\|_{\infty} \leq C_{\sigma}\|\widetilde{g}\|_{1}\|f\|_{\infty} .
$$

By (43), (44), and the Riesz-Thorin interpolation Theorem we further obtain for $f \in L^{p}\left(\mathbb{B}_{t}^{n}, d \mu_{\sigma, t}\right)$ and $g \in L^{1}\left(\mathbb{B}_{t}^{n}, d \mu_{\sigma, t}\right)$ the inequality

$$
\|f * g\|_{p} \leq C_{\sigma}\|\widetilde{g}\|_{1}\|f\|_{p}
$$

To obtain a Young's inequality for the $(\sigma, t)$-convolution we consider only the case $\sigma \leq 2-n$.

Theorem 1 Let $\sigma \leq 2-n, 1 \leq p, q, r \leq \infty, \frac{1}{p}+\frac{1}{q}=1+\frac{1}{r}, s=1-\frac{q}{r}, f \in L^{p}\left(\mathbb{B}_{t}^{n}, d \mu_{\sigma, t}\right)$ and $g \in L^{q}\left(\mathbb{B}_{t}^{n}, d \mu_{\sigma, t}\right)$. Then

$$
\|f * g\|_{r} \leq 4^{\frac{2-n-\sigma}{2}}\|\widetilde{g}\|_{q}^{1-s}\|g\|_{q}^{s}\|f\|_{p}
$$

where $\widetilde{g}(x):=\underset{y \in \mathbb{B}_{t}^{n}}{\operatorname{ess} \sup } g(\operatorname{gyr}[y, x] x)$, for any $x \in \mathbb{B}_{t}^{n}$.

Proof: First case: $p=1$ and $r=q$. The following estimate is used in the proof:

$$
\left.\left.j_{x}(y) j_{x}(x) \leq 4^{\frac{2-n-\sigma}{2}}, \forall x, y \in \mathbb{B}_{t}^{n}, \forall \sigma \in\right]-\infty, 2-n\right] .
$$

Then, considering $K=\|f * g\|_{q}$ we have 


$$
\begin{aligned}
K & =\left(\int_{\mathbb{B}_{t}^{n}}\left|\int_{\mathbb{B}_{t}^{n}} f(y) \tau_{x} g(-y) j_{x}(x) d \mu_{\sigma, t}(y)\right|^{q} d \mu_{\sigma, t}(x)\right)^{1 / q} \\
& \leq \int_{\mathbb{B}_{t}^{n}}|f(y)|\left(\int_{\mathbb{B}_{t}^{n}}\left|\tau_{x} g(-y) j_{x}(x)\right|^{q} d \mu_{\sigma, t}(x)\right)^{1 / q} d \mu_{\sigma, t}(y) \quad \text { (Minkowski's inequality) } \\
& =\int_{\mathbb{B}_{t}^{n}}|f(y)|\left(\int_{\mathbb{B}_{t}^{n}}\left|g(x \oplus(-y)) j_{x}(y) j_{x}(x)\right|^{q} d \mu_{\sigma, t}(x)\right)^{1 / q} d \mu_{\sigma, t}(y) \quad \text { by (17)) } \\
& =\int_{\mathbb{B}_{t}^{n}}|f(y)|\left(\int_{\mathbb{B}_{t}^{n}}\left|g(\operatorname{gyr}[x,-y]((-y) \oplus x)) j_{y}(x) j_{y}(y)\right|^{q} d \mu_{\sigma, t}(x)\right)^{1 / q} d \mu_{\sigma, t}(y)
\end{aligned}
$$

(by $(5),(21),(20))$

$$
=\int_{\mathbb{B}_{t}^{n}}|f(y)|\left(\int_{\mathbb{B}_{t}^{n}}\left|g(\operatorname{gyr}[y \oplus z,-y] z) j_{y}(y \oplus z) j_{y}(y)\right|^{q}\left(j_{y}(-z)\right)^{2} d \mu_{\sigma, t}(z)\right)^{1 / q} d \mu_{\sigma, t}(y)
$$

(change of variables $z=(-y) \oplus x \Leftrightarrow x=y \oplus z$, and (40))

$$
=\int_{\mathbb{B}_{t}^{n}}|f(y)|\left(\int_{\mathbb{B}_{t}^{n}}|g(\operatorname{gyr}[y \oplus z,-y] z)|^{q}\left(j_{y}(y \oplus z) j_{y}(y)\right)^{q-1} j_{y}(-z) j_{y}(y) d \mu_{\sigma, t}(z)\right)^{1 / q} d \mu_{\sigma, t}(y)
$$

(by (26))

$$
\begin{aligned}
& \left.\leq 4^{\frac{2-n-\sigma}{2}} \int_{\mathbb{B}_{t}^{n}}|f(y)|\left(\int_{\mathbb{B}_{t}^{n}}|g(\operatorname{gyr}[y, z] z)|^{q} d \mu_{\sigma, t}(z)\right)^{1 / q} d \mu_{\sigma, t}(y) \quad \text { (by }(9),(46)\right) \\
& =4^{\frac{2-n-\sigma}{2}}\|f\|_{1}\|\widetilde{g}\|_{q} .
\end{aligned}
$$

Second case: $r=\infty$ and $\frac{1}{p}+\frac{1}{q}=1$. Then,

$$
\begin{aligned}
& \|f * g\|_{\infty} \leq \sup _{x \in \mathbb{B}_{t}^{n}} \int_{\mathbb{B}_{t}^{n}}\left|f(y) \tau_{x} g(-y) j_{x}(x)\right| d \mu_{\sigma, t}(y) \\
& \leq \sup _{x \in \mathbb{B}_{t}^{n}}\|f\|_{p}\left(\int_{\mathbb{B}_{t}^{n}}\left|g(x \oplus(-y)) j_{x}(y) j_{x}(x)\right|^{q} d \mu_{\sigma, t}(y)\right)^{1 / q} \\
& =\|f\|_{p} \sup _{x \in \mathbb{B}_{t}^{n}}\left(\int_{\mathbb{B}_{t}^{n}}\left|g(z) j_{x}(x \oplus(-z)) j_{x}(x)\right|^{q}\left(j_{x}(z)\right)^{2} d \mu_{\sigma, t}(z)\right)^{1 / q} \\
& \text { (change of variables } z=x \oplus(-y) \Leftrightarrow y=x \oplus(-z) \text {, and (40)) } \\
& =\|f\|_{p} \sup _{x \in \mathbb{B}_{t}^{n}}\left(\int_{\mathbb{B}_{t}^{n}}|g(z)|^{q}\left(j_{x}(x \oplus(-z)) j_{x}(x)\right)^{q-1} j_{x}(z) j_{x}(x) d \mu_{\sigma, t}(z)\right)^{1 / q} \\
& \text { (by (46)) } \\
& \leq 4^{\frac{2-n-\sigma}{2}}\|f\|_{p}\|g\|_{q} \quad(\text { by }(26)) \text {. }
\end{aligned}
$$

General case: Let $1 \leq q \leq \infty$ and $g \in L^{q}\left(\mathbb{B}_{t}^{n}, d \mu_{\sigma, t}\right)$. Considering the linear operator $T$ defined by $T_{g}(f)=f * g$ we have by the previous cases

$$
\left\|T_{g}(f)\right\|_{q} \leq 4^{\frac{2-n-\sigma}{2}}\|\widetilde{g}\|_{q}\|f\|_{1}, \quad \text { i.e. } \quad T: L^{1} \rightarrow L^{q}
$$


and

$$
\left\|T_{g}(f)\right\|_{\infty} \leq 4^{\frac{2-n-\sigma}{2}}\|g\|_{q}\|f\|_{p}, \quad \text { i.e. } \quad T: L^{p} \rightarrow L^{\infty}
$$

with $1 / p+1 / q=1$. By the Riesz-Thorin interpolation theorem we obtain

$$
\left\|T_{g}(f)\right\|_{r} \leq 4^{\frac{2-n-\sigma}{2}}\|\widetilde{g}\|_{q}^{1-s}\|g\|_{q}^{s}\|f\|_{p}
$$

with $\frac{1}{p}+\frac{1}{q}=1+\frac{1}{r}$ and $s=1-\frac{q}{r}$.

Corollary 3 Let $\sigma \leq 2-n, 1 \leq p, q, r \leq \infty, \frac{1}{p}+\frac{1}{q}=1+\frac{1}{r}, f \in L^{p}\left(\mathbb{B}_{t}^{n}, d \mu_{\sigma, t}\right)$ and $g \in L^{q}\left(\mathbb{B}_{t}^{n}, d \mu_{\sigma, t}\right)$ a radial function. Then,

$$
\|f * g\|_{r} \leq 4^{\frac{2-n-\sigma}{2}}\|g\|_{q}\|f\|_{p}
$$

Remark 1 For $\sigma=2-n$ and taking the limit $t \rightarrow+\infty$ in (45) we recover Young's inequality for the Euclidean convolution in $\mathbb{R}^{n}$ since in the limit $\widetilde{g}=g$.

Another important property of the Euclidean convolution is its translation invariance. In the hyperbolic case the convolution is gyrotranslation invariant.

Theorem 2 The $(\sigma, t)$-convolution is gyrotranslation invariant, i.e.,

$$
\tau_{a}(f * g)(x)=\left(\tau_{a} f(\cdot) * g(\operatorname{gyr}[-a, x] \cdot)\right)(x) .
$$

Proof: By (17), (42), and (24) we have

$$
\begin{aligned}
\tau_{a}(f * g)(x) & =(f * g)((-a) \oplus x) j_{a}(x) \\
& =\int_{\mathbb{B}_{t}^{n}} f(y) \tau_{(-a) \oplus x} g(-y) j_{(-a) \oplus x}((-a) \oplus x) j_{a}(x) d \mu_{\sigma, t}(y) \\
& =\int_{\mathbb{B}_{t}^{n}} f(y) \tau_{(-a) \oplus x} g(-y) j_{x}(x) d \mu_{\sigma, t}(y) .
\end{aligned}
$$

From (29) we can easily conclude that

$$
\tau_{b \oplus a} f(x)=\tau_{b} \tau_{a} f(\operatorname{gyr}[b, a] x)
$$

since gyr $[a, b] \operatorname{gyr}[b, a]=I$ by (8). Therefore, applying (49) we obtain

$$
\begin{aligned}
\tau_{a}(f * g)(x) & =\int_{\mathbb{B}_{t}^{n}} f(y) \tau_{(-a) \oplus x} g(-y) j_{x}(x) d \mu_{\sigma, t}(y) \\
& =\int_{\mathbb{B}_{t}^{n}} f(y) \tau_{-a} \tau_{x} g(-\operatorname{gyr}[-a, x] y) j_{x}(x) d \mu_{\sigma, t}(y) \\
& \left.=\int_{\mathbb{B}_{t}^{n}} \tau_{a} f(y) \tau_{x} g(-\operatorname{gyr}[-a, x] y) j_{x}(x) d \mu_{\sigma, t}(y) \quad \text { (by }(39)\right) \\
& =\left(\tau_{a} f(\cdot) * g(\operatorname{gyr}[-a, x] \cdot)\right)(x) .
\end{aligned}
$$

In Theorem 2 if $g$ is a radial function then we obtain the translation invariant property $\tau_{a}(f * g)=$ $\left(\tau_{a} f\right) * g$. The next theorem shows that the $(\sigma, t)$-convolution is gyroassociative. 
Theorem 3 If $f, g, h \in L^{1}\left(\mathbb{B}_{t}^{n}, d \mu_{\sigma, t}\right)$ then

$$
\left(f *_{a}\left(g *_{x} h\right)\right)(a)=\left(\left(\left(f(x) *_{y} g(\operatorname{gyr}[a,-(y \oplus x)] \operatorname{gyr}[y, x] x)\right)(y)\right) *_{a} h(y)\right)(a)
$$

Proof: The result of the theorem is proved in the following chain of equations:

$$
\begin{aligned}
& (f *(g * h))(a)=\int_{\mathbb{B}_{t}^{n}} f(x) \tau_{a}(h * g)(-x) j_{a}(a) d \mu_{\sigma, t}(x) \quad(g * h=h * g) \\
& =\int_{\mathbb{B}_{t}^{n}} f(x)(h * g)(a \oplus(-x)) j_{a}(x) j_{a}(a) d \mu_{\sigma, t}(x) \quad(\text { by }(18)) \\
& =\int_{\mathbb{B}_{t}^{n}} f(x) \int_{\mathbb{B}_{t}^{n}} h(y) \tau_{a \oplus(-x)} g(-y) j_{a \oplus(-x)}(a \oplus(-x)) j_{a}(x) d \mu_{\sigma, t}(y) j_{a}(a) d \mu_{\sigma, t}(x)
\end{aligned}
$$

$$
=\int_{\mathbb{B}_{t}^{n}} f(x) \int_{\mathbb{B}_{t}^{n}} h(y) \tau_{a} \tau_{-x} g(-\operatorname{gyr}[a,-x] y) j_{x}(x) d \mu_{\sigma, t}(y) j_{a}(a) d \mu_{\sigma, t}(x)
$$

$$
=\int_{\mathbb{B}_{t}^{n}} f(x) \int_{\mathbb{B}_{t}^{n}} \tau_{-a} h(y) \tau_{-x} g(-\operatorname{gyr}[a,-x] y) j_{x}(x) d \mu_{\sigma, t}(y) j_{a}(a) d \mu_{\sigma, t}(x)
$$

$$
=\int_{\mathbb{B}_{t}^{n}} f(x) \int_{\mathbb{B}_{t}^{n}} h(a \oplus y) j_{-a}(y) g(-\operatorname{gyr}[a,-x](x \oplus y)) j_{-x}(y) j_{x}(x) d \mu_{\sigma, t}(y) j_{a}(a) d \mu_{\sigma, t}(x)
$$

(by (18))

$$
=\int_{\mathbb{B}_{t}^{n}} f(x) \int_{\mathbb{B}_{t}^{n}} \tau_{a} h(-y) \tau_{x} g(\operatorname{gyr}[a,-x] y) j_{x}(x) d \mu_{\sigma, t}(y) j_{a}(a) d \mu_{\sigma, t}(x)
$$

(by change $y \mapsto-y,(19),(17)$ )

$$
\begin{aligned}
& =\int_{\mathbb{B}_{t}^{n}} \tau_{a} h(-y) \int_{\mathbb{B}_{t}^{n}} f(x) \tau_{x} g(\operatorname{gyr}[a,-x] y) j_{x}(x) d \mu_{\sigma, t}(x) j_{a}(a) d \mu_{\sigma, t}(y) \quad \text { (Fubini) } \\
& =\int_{\mathbb{B}_{t}^{n}} \tau_{a} h(-y) \int_{\mathbb{B}_{t}^{n}} f(x) \tau_{y} g(-\operatorname{gyr}[a,-(y \oplus x)] \operatorname{gyr}[y, x] x) j_{y}(y) d \mu_{\sigma, t}(x) j_{a}(a) d \mu_{\sigma, t}(y)
\end{aligned}
$$

$=\left(\left(\left(f(x) *_{y} g(\operatorname{gyr}[a,-(y \oplus x)] \operatorname{gyr}[y, x] x)\right)(y)\right) *_{a} h(y)\right)(a)$.

Corollary 4 If $f, g, h \in L^{1}\left(\mathbb{B}_{t}^{n}, d \mu_{\sigma, t}\right)$ and $g$ is a radial function then the $(\sigma, t)$-convolution is associative. i.e.,

$$
f *(g * h)=(f * g) * h .
$$

From Theorem 3 we see that the $(\sigma, t)$-convolution is associative up to a gyration of the argument of the function $g$. However, if $g$ is a radial function then the corresponding gyration is trivial (that is, it is the identity map) and therefore the $(\sigma, t)$-convolution becomes associative. Moreover, in the limit $t \rightarrow+\infty$ gyrations reduce to the identity, so that formula (50) becomes associative in the Euclidean case. If we denote by $L_{R}^{1}\left(\mathbb{B}_{t}^{n}, d \mu_{\sigma, t}\right)$ the subspace of $L^{1}\left(\mathbb{B}_{t}^{n}, d \mu_{\sigma, t}\right)$ consisting of radial functions then, for $\sigma<1, L_{R}^{1}\left(\mathbb{B}_{t}^{n}, d \mu_{\sigma, t}\right)$ is a commutative associative Banach algebra under the $(\sigma, t)$-convolution. 


\section{$5 \quad$ Eigenfunctions of $\Delta_{\sigma, t}$}

We begin by defining the $(\sigma, t)$-hyperbolic plane waves which are the relativistic counterpart of the Euclidean plane waves and proceed with the study of its properties.

Definition 3 For $\lambda \in \mathbb{C}, \xi \in \mathbb{S}^{n-1}$, and $x \in \mathbb{B}_{t}^{n}$ we define the functions $e_{\lambda, \xi ; t}$ by

$$
e_{\lambda, \xi ; t}(x)=\frac{\left(1-\frac{\|x\|^{2}}{t^{2}}\right)^{\frac{1-\sigma+\mathrm{i} \lambda t}{2}}}{\left(\left\|\xi-\frac{x}{t}\right\|^{2}\right)^{\frac{n-1+\mathrm{i} \lambda t}{2}}} .
$$

Proposition 5 The function $e_{\lambda, \xi ; t}$ is an eigenfunction of $\Delta_{\sigma, t}$ with eigenvalue $-\frac{(1-\sigma)^{2}}{t^{2}}-\lambda^{2}$.

Proof: Since

$$
\begin{aligned}
& \Delta e_{\lambda, \xi ; t}(x)=\left((1-\sigma+\mathrm{i} \lambda t)(-1-\sigma+\mathrm{i} \lambda t)\left(1-\frac{\|x\|^{2}}{t^{2}}\right)^{-2} \frac{\|x\|^{2}}{t^{4}}-(1-\sigma+\mathrm{i} \lambda t) \frac{n}{t^{2}}\left(1-\frac{\|x\|^{2}}{t^{2}}\right)^{-1}\right. \\
& \left.+\frac{(1+\mathrm{i} \lambda t)}{t^{2}} \frac{(n-1+\mathrm{i} \lambda t)}{\left\|\xi-\frac{x}{t}\right\|^{2}}+\frac{2}{t^{2}}(1-\sigma+\mathrm{i} \lambda t)(n-1+\mathrm{i} \lambda t)\left(1-\frac{\|x\|^{2}}{t^{2}}\right)^{-1} \frac{\|x\|^{2}}{t^{2}}-\frac{\langle x, \xi\rangle}{t}\right) e_{\lambda, \xi ; t}(x)
\end{aligned}
$$

and

$$
\nabla e_{\lambda, \xi ; t}(x)=\left(-(1-\sigma+\mathrm{i} \lambda t)\left(1-\frac{\|x\|^{2}}{t^{2}}\right)^{-1} \frac{x}{t^{2}}-(n-1+\mathrm{i} \lambda t) \frac{\frac{x}{t^{2}}-\frac{\xi}{t}}{\left\|\xi-\frac{x}{t}\right\|^{2}}\right) e_{\lambda, \xi ; t}(x)
$$

then we have, by straightforward computations,

$$
\begin{aligned}
\Delta_{\sigma, t} e_{\lambda, \xi ; t}(x)= & \left(1-\frac{\|x\|^{2}}{t^{2}}\right)^{2} \Delta e_{\lambda, \xi ; t}(x)+\left(1-\frac{\|x\|^{2}}{t^{2}}\right)\left(-\frac{2 \sigma}{t^{2}}\left\langle x, \nabla e_{\lambda, \xi ; t}(x)\right\rangle\right. \\
& \left.+\frac{\sigma(2-n-\sigma)}{t^{2}} e_{\lambda, \xi ; t}(x)\right) \\
= & -\left(\frac{(1-\sigma)^{2}}{t^{2}}+\lambda^{2}\right) e_{\lambda, \xi ; t}(x) .
\end{aligned}
$$

The $(\sigma, t)$-hyperbolic plane waves $e_{\lambda, \xi ; t}(x)$ converge in the limit $t \rightarrow+\infty$ to the Euclidean plane waves $\mathrm{e}^{\mathrm{i}\langle x, \eta\rangle}$, where $\eta=\lambda \xi \in \mathbb{R}^{n}$, for $\lambda \in \mathbb{R}$. For $x \in \mathbb{R}^{n}$, choose $t_{0}>0$ such that $x \in B_{t_{0}}^{n}$. Then $x \in \mathbb{B}_{t}^{n}$ for all $t>t_{0}$ and, moreover,

$$
\begin{aligned}
e_{\lambda, \xi ; t}(x) & =\left(\frac{t^{2}+\|x\|^{2}}{t^{2}-\|x\|^{2}}-\frac{2 t\langle x, \xi\rangle}{t^{2}-\|x\|^{2}}\right)^{-\frac{(n-1+\mathrm{i} \lambda t)}{2}}\left(1-\frac{\|x\|^{2}}{t^{2}}\right)^{\frac{2-n-\sigma}{2}} \\
& =\left(\frac{t^{2}+\|x\|^{2}}{t^{2}-\|x\|^{2}}-\frac{2}{t}\langle y, \xi\rangle\right)^{-\frac{(n-1+\mathrm{i} \lambda t)}{2}}\left(1-\frac{\|x\|^{2}}{t^{2}}\right)^{\frac{2-n-\sigma}{2}}, \quad t>t_{0},
\end{aligned}
$$

where $y=\frac{t^{2}}{t^{2}-\|x\|^{2}} x \in \mathbb{R}^{n}$. Letting $t \rightarrow+\infty$ we observe that $y$ tends to $x$ and

$$
\lim _{t \rightarrow+\infty} e_{\lambda, \xi ; t}(x)=\lim _{t \rightarrow+\infty}\left[\left(1-\frac{2\langle y, \eta\rangle}{t}\right)^{t}\right]^{\frac{-\mathrm{i} \lambda}{2}}=\mathrm{e}^{\mathrm{i}\langle x, \eta\rangle}
$$


with $\eta=\lambda \xi \in \mathbb{R}^{n}$. Moreover, the eigenvalues of $\Delta_{\sigma, t}$ converge to $-\lambda^{2}=-\|\eta\|^{2}$, which are the eigenvalues of the Laplace operator in $\mathbb{R}^{n}$ associated to the eigenfunctions $\mathrm{e}^{\mathrm{i}\langle x, \eta\rangle}$. In the Euclidean case given two eigenfunctions $\mathrm{e}^{\mathrm{i}\langle x, \lambda \xi\rangle}$ and $\mathrm{e}^{\mathrm{i}\langle x, \gamma \omega\rangle}, \lambda, \gamma \in \mathbb{R}, \xi, \omega \in \mathbb{S}^{n-1}$ of the Laplace operator with eigenvalues $-\lambda^{2}$ and $-\gamma^{2}$ respectively, the product of the two eigenfunctions is again an eigenfunction of the Laplace operator with eigenvalue $-\left(\lambda^{2}+\gamma^{2}+2 \lambda \gamma\langle\xi, \omega\rangle\right)$. Indeed,

$$
\Delta\left(\mathrm{e}^{\mathrm{i}\langle x, \lambda \xi\rangle} \mathrm{e}^{\mathrm{i}\langle x, \gamma \omega\rangle}\right)=-\|\lambda \xi+\gamma \omega\|^{2} \mathrm{e}^{\mathrm{i}\langle x, \lambda \xi+\gamma \omega\rangle}=-\left(\lambda^{2}+\gamma^{2}+2 \lambda \gamma\langle\xi, \omega\rangle\right) \mathrm{e}^{\mathrm{i}\langle x, \lambda \xi+\gamma \omega\rangle} .
$$

Unfortunately, in the hyperbolic case this is no longer true in general. The only exception is the case $n=1$ and $\sigma=1$ as the next proposition shows.

Proposition 6 For $n \geq 2$ the product of two eigenfunctions of $\Delta_{\sigma, t}$ is not an eigenfunction of $\Delta_{\sigma, t}$ and for $n=1$ the product of two eigenfunctions of $\Delta_{\sigma, t}$ is an eigenfunction of $\Delta_{\sigma, t}$ only in the case $\sigma=1$.

Proof: For $n \geq 1, f, g \in C^{2}\left(\mathbb{B}_{t}^{n}\right)$ the Leibniz rule for $\Delta_{\sigma, t}$ is given by

$$
\Delta_{\sigma, t}(f g)=\left(\Delta_{\sigma, t} f\right) g+f\left(\Delta_{\sigma, t} g\right)+2\left(1-\frac{\|x\|^{2}}{t^{2}}\right)^{2}\langle\nabla f, \nabla g\rangle-\frac{\sigma(2-n-\sigma)}{t^{2}}\left(1-\frac{\|x\|^{2}}{t^{2}}\right) f g .
$$

Considering $e_{\lambda, \xi ; t}$ and $e_{\gamma, \omega ; t}$ two eigenfunctions of $\Delta_{\sigma, t}$ with eigenvalues $-\frac{(1-\sigma)^{2}}{t^{2}}-\lambda^{2}$ and $-\frac{(1-\sigma)^{2}}{t^{2}}-\gamma^{2}$ respectively, we obtain by (53) and Proposition 5

$$
\begin{aligned}
& \Delta_{\sigma, t}\left(e_{\lambda, \xi ; t}(x) e_{\gamma, \omega ; t}(x)\right)=\left[-\frac{2(1-\sigma)^{2}}{t^{2}}-\lambda^{2}-\gamma^{2}+2\left((1-\sigma+\mathrm{i} \lambda t)(1-\sigma+\mathrm{i} \gamma t) \frac{\|x\|^{2}}{t^{2}}\right.\right. \\
& +(1-\sigma+\mathrm{i} \lambda t)(n-1+\mathrm{i} \gamma t)\left(1-\frac{\|x\|^{2}}{t^{2}}\right) \frac{1}{t^{4}} \frac{\|x\|^{2}-t\langle x, \omega\rangle}{\left\|\frac{x}{t}-\omega\right\|^{2}}+(1-\sigma+\mathrm{i} \gamma t)(n-1+\mathrm{i} \lambda t) \\
& \times\left(1-\frac{\|x\|^{2}}{t^{2}}\right) \frac{1}{t^{4}} \frac{\|x\|^{2}-t\langle\xi, \omega\rangle}{\left\|\frac{x}{t}-\xi\right\|^{2}}+\left[(n-1+\mathrm{i} \lambda t)(n-1+\mathrm{i} \gamma t)\left(1-\frac{\|x\|^{2}}{t^{2}}\right)^{2} \frac{1}{t^{4}}\right. \\
& \left.\left.\times \frac{\|x\|^{2}-t\langle x, \omega\rangle-t\langle x, \xi\rangle+t^{2}\langle\xi, \omega\rangle}{\left\|\frac{x}{t}-\xi\right\|^{2}\left\|\frac{x}{t}-\omega\right\|^{2}}\right)-\frac{\sigma(2-n-\sigma)}{t^{2}}\left(1-\frac{\|x\|^{2}}{t^{2}}\right)\right] e_{\lambda, \xi ; t}(x) e_{\gamma, \omega ; t}(x) .
\end{aligned}
$$

Therefore, for $n \geq 2$ and $\sigma \in \mathbb{R}$, the product of two eigenfunctions of $\Delta_{\sigma, t}$ is not an eigenfunction of $\Delta_{\sigma, t}$. For $n=1$ the previous formula reduces to

$$
\Delta_{\sigma, t}\left(e_{\lambda, \xi ; t}(x) e_{\gamma, \omega ; t}(x)\right)=\left[-\lambda^{2}-\gamma^{2}-2 \lambda \gamma \xi \omega+2\left(\frac{\sigma-1}{t^{2}}\left(1-\frac{x^{2}}{t^{2}}+\mathrm{i} \gamma x \omega+\mathrm{i} \lambda x \xi\right)\right)\right] e_{\lambda, \xi ; t}(x) e_{\gamma, \omega ; t}(x) .
$$

For $\sigma=1$, which corresponds to the case $\sigma=2-n$ for $n=1$, we further obtain

$$
\Delta_{\sigma, t}\left(e_{\lambda, \xi ; t}(x) e_{\gamma, \omega ; t}(x)\right)=-\left(\lambda^{2}+\gamma^{2}+2 \lambda \gamma \xi \omega\right) e_{\lambda, \xi ; t}(x) e_{\gamma, \omega ; t}(x) .
$$

Thus, only in the case $n=1$ and $\sigma=1$ the product of two eigenfunctions of $\Delta_{\sigma, t}$ is an eigenfunction of $\Delta_{\sigma, t}$. 
We remark that in the case when $n=1$ and $\sigma=1$ the hyperbolic plane waves (51) are independent of $\xi$ since they reduce to

$$
e_{\lambda ; t}(x)=\left(\frac{1+\frac{x}{t}}{1-\frac{x}{t}}\right)^{\frac{\mathrm{i} \lambda t}{2}}
$$

and, therefore, the exponential law is valid, i.e., $e_{\lambda ; t}(x) e_{\gamma ; t}(x)=e_{\lambda+\gamma ; t}(x)$.

For the special case when $\sigma=2-n$ the $(\sigma, t)$-hyperbolic plane waves $(51)$ defined on $\mathbb{B}_{t}^{n}$ become the hyperbolic plane waves defined on the hyperboloid $H_{n}^{+}$of radius $t$ embedded in $\mathbb{R}^{n+1}$ (see [3]). Considering the coordinates $z=\left(z_{0}, \mathbf{z}\right) \in H_{n}^{+}$where $z_{0}=t \cosh (\alpha)$ and $\mathbf{z}=t \sinh (\alpha) n$, with $\alpha \in \mathbb{R}^{+}$ and $n \in \mathbb{S}^{n-1}$, and the change of variables given by $\cosh (\alpha)=\frac{t^{2}+\|x\|^{2}}{t^{2}-\|x\|^{2}}$ and $\sinh (\alpha)=\frac{2 t\|x\|}{t^{2}-\|x\|^{2}}$, with $x \in \mathbb{B}_{t}^{n}$, we obtain the functions

$$
\begin{aligned}
\Phi_{\lambda, \xi}(z) & =(\cosh (\alpha)-\sinh (\alpha)\langle n, \xi\rangle)^{-\frac{n-1+\mathrm{i} \lambda t}{2}} \quad(n=x /\|x\|) \\
& =\left(\frac{z_{0}-\langle\mathbf{z}, \xi\rangle}{t}\right)^{-\frac{n-1+\mathrm{i} \lambda t}{2}} .
\end{aligned}
$$

These functions are also known as Shapiro functions and they form a complete orthogonal Dirac basis on the hyperboloid (see [3]), satisfying:

$$
\int_{H_{n}^{+}} \Phi_{\lambda, \xi}(z) \overline{\Phi_{\lambda^{\prime}, \xi^{\prime}}(z)} d \mu(z)=(2 \pi)^{n} N^{(n)}(\lambda t) \delta\left(\frac{\lambda}{2} \xi-\frac{\lambda^{\prime}}{2} \xi^{\prime}\right)
$$

where

$$
d \mu(z)=t^{n} \sinh ^{n-1}(\alpha) d \alpha d n \quad \text { and } \quad N^{(n)}(\lambda t)=\left|\frac{\Gamma\left(\frac{\mathrm{i} \lambda t}{2}\right)}{\Gamma\left(\frac{n-1}{2}+\frac{\mathrm{i} \lambda t}{2}\right)}\right|^{2}\left(\frac{\lambda t}{2}\right)^{n-1} .
$$

Therefore, in the case $\sigma=2-n$, since $d \mu(z)=2^{n} d \mu_{\sigma, t}(x)$ under the change of variables above, and $\delta(\lambda / 2)=2^{n} \delta(\lambda)$ by the scaling property of the $\delta$-function, it is easy to see that the functions $e_{\lambda, \xi ; t}$ form a complete orthogonal Dirac basis on $\mathbb{B}_{t}^{n}$ satisfying

$$
\int_{\mathbb{B}_{t}^{n}} e_{\lambda, \xi ; t}(x) \overline{e_{\lambda^{\prime}, \xi^{\prime} ; t}(x)} d \mu_{\sigma, t}(x)=(2 \pi)^{n} N^{(n)}(\lambda t) \delta\left(\lambda \xi-\lambda^{\prime} \xi^{\prime}\right)
$$

In the Euclidean case the translation of the Euclidean plane waves $e^{\mathrm{i}\langle x, \lambda \xi\rangle}$ decomposes into the product of two plane waves one being a modulation. In the hyperbolic case we have an analogous result for the $(\sigma, t)$-translation of the $(\sigma, t)$-hyperbolic plane waves but it appears a Möbius transformation acting on $\mathbb{S}^{n-1}$ as the next proposition shows.

Proposition 7 The $(\sigma, t)$-translation of $e_{\lambda, \xi ; t}$ admits the factorisation

$$
\tau_{a} e_{\lambda, \xi ; t}(x)=j_{a}(0) e_{\lambda, \xi ; t}(-a) e_{\lambda, a \oplus \xi ; t}(x)
$$


Proof: By (16), (1) and (15) we have

$$
\begin{aligned}
\tau_{a} e_{\lambda, \xi ; t}(x) & =e_{\lambda, \xi ; t}((-a) \oplus x) j_{a}(x) \\
& =\frac{\left(1-\frac{\|\varphi-a(x)\|^{2}}{t^{2}}\right)^{\frac{1-\sigma+i \lambda t}{2}}\left(\frac{1-\frac{\|a\|^{2}}{t^{2}}}{\left\|1+\frac{a x}{t^{2}}\right\|}\right)^{\frac{n+\sigma-2}{2}}}{\left\|\xi-\frac{\varphi-a(x)}{t}\right\|^{n-1+\mathrm{i} \lambda t}} \\
& =\frac{\left[\left(1-\frac{\|a\|^{2}}{t^{2}}\right)\left(1-\frac{\|x\|^{2}}{t^{2}}\right)\left\|1+\frac{a x}{t^{2}}\right\|^{-2}\right]^{\frac{1-\sigma+\mathrm{i} \lambda t}{2}}}{\left[\left\|\xi-\frac{a}{t}\right\|\left\|\left(\xi+\frac{a}{t}\right)\left(1-\frac{a \xi}{t}\right)^{-1}-\frac{x}{t}\right\|\left\|1+\frac{a x}{t^{2}}\right\|^{-1}\right]^{n-1+\mathrm{i} \lambda t}}\left(\frac{1-\frac{\|a\|^{2}}{t^{2}}}{\left\|1+\frac{a x}{t^{2}}\right\|}\right)^{\frac{n+\sigma-2}{2}} \\
& =\frac{\left(1-\frac{\|a\|^{2}}{t^{2}}\right)^{\frac{1-\sigma+\mathrm{i} \lambda t}{2}}}{\left\|\xi+\frac{a}{t}\right\|^{n-1+\mathrm{i} \lambda t}} \frac{\|x\|^{2}}{\left\|\left(\frac{a}{t}+\xi\right)\left(1-\frac{a \xi}{t}\right)^{-1}-\frac{x}{t}\right\|^{n-1+\mathrm{i} \lambda t}}\left(1-\frac{\|a\|^{2}}{t^{2}}\right)^{\frac{n+\sigma-2}{2}} \\
& =e_{\lambda, \xi ; t}(-a) e_{\lambda, a \oplus \xi ; t}(x) j_{a}(0) .
\end{aligned}
$$

Remark 2 The fractional linear mappings $a \oplus \xi=\varphi_{a}(\xi), a \in \mathbb{B}_{t}^{n}, \xi \in \mathbb{S}^{n-1}$ are given by

$$
a \oplus \xi=\left(\frac{a}{t}+\xi\right)\left(1-\frac{a}{t} \xi\right)^{-1}=\frac{2\left(1+\frac{1}{t}\langle a, \xi\rangle\right) \frac{a}{t}+\left(1-\frac{\|a\|^{2}}{t^{2}}\right) \xi}{1+\frac{2}{t}\langle a, \xi\rangle+\frac{\|a\|^{2}}{t^{2}}}
$$

and map $\mathbb{S}^{n-1}$ onto itself for any $t>0$ and $a \in \mathbb{B}_{t}^{n}$. In the limit $t \rightarrow+\infty$ they reduce to the identity mapping on $\mathbb{S}^{n-1}$. Therefore, formula (56) converges in the limit to the well-known formula in the Euclidean case

$$
\mathrm{e}^{\mathrm{i}\langle-a+x, \lambda \xi\rangle}=\mathrm{e}^{\mathrm{i}\langle-a, \lambda \xi\rangle} \mathrm{e}^{\mathrm{i}\langle x, \lambda \xi\rangle}, \quad a, x, \lambda \xi \in \mathbb{R}^{n} .
$$

Now we study the radial eigenfunctions of $\Delta_{\sigma, t}$, which are called $(\sigma, t)$-spherical functions.

Definition 4 For each $\lambda \in \mathbb{C}$, we define the elementary $(\sigma, t)$-spherical function $\phi_{\lambda ; t}$ by

$$
\phi_{\lambda ; t}(x)=\int_{\mathbb{S}^{n-1}} e_{\lambda, \xi ; t}(x) d \sigma(\xi), \quad x \in \mathbb{B}_{t}^{n} .
$$

Using (A.1) in Appendix A and then (A.3) in Appendix A we can write $\phi_{\lambda ; t}$ as

$$
\begin{aligned}
\phi_{\lambda ; t}(x) & =\left(1-\frac{\|x\|^{2}}{t^{2}}\right)^{\frac{1-\sigma+\mathrm{i} \lambda t}{2}}{ }_{2} F_{1}\left(\frac{n-1+\mathrm{i} \lambda t}{2}, \frac{1+\mathrm{i} \lambda t}{2} ; \frac{n}{2} ; \frac{\|x\|^{2}}{t^{2}}\right) \\
& =\left(1-\frac{\|x\|^{2}}{t^{2}}\right)^{\frac{1-\sigma-\mathrm{i} \lambda t}{2}}{ }_{2} F_{1}\left(\frac{n-1-\mathrm{i} \lambda t}{2}, \frac{1-\mathrm{i} \lambda t}{2} ; \frac{n}{2} ; \frac{\|x\|^{2}}{t^{2}}\right) .
\end{aligned}
$$

Therefore, $\phi_{\lambda ; t}$ is a radial function that satisfies $\phi_{\lambda ; t}=\phi_{-\lambda ; t}$ i.e., $\phi_{\lambda ; t}$ is an even function of $\lambda \in \mathbb{C}$. Putting $\|x\|=t \tanh s$, with $s \in \mathbb{R}^{+}$, and applying (A.4) in Appendix 1 we have the following relation 
between $\phi_{\lambda ; t}$ and the Jacobi functions (cf. (B.2) in Appendix B):

$$
\begin{aligned}
\phi_{\lambda ; t}(t \tanh s) & =(\cosh s)^{n-2+\sigma}{ }_{2} F_{1}\left(\frac{n-1-\mathrm{i} \lambda t}{2}, \frac{n-1+\mathrm{i} \lambda t}{2} ; \frac{n}{2} ;-\sinh ^{2}(s)\right) \\
& =(\cosh s)^{n-2+\sigma} \varphi_{\lambda t}^{\left(\frac{n}{2}-1, \frac{n}{2}-1\right)}(s) .
\end{aligned}
$$

The following theorem characterises all $(\sigma, t)$-spherical functions.

Theorem 4 The function $\phi_{\lambda ; t}$ is a $(\sigma, t)$-spherical function with eigenvalue $-\frac{(1-\sigma)^{2}}{t^{2}}-\lambda^{2}$. Moreover, if we normalize $(\sigma, t)$-spherical functions $\phi_{\lambda ; t}$ such that $\phi_{\lambda ; t}(0)=1$, then all $(\sigma, t)$-spherical functions are given by $\phi_{\lambda ; t}$.

Proof: By Proposition 5 it is easy to see that $\phi_{\lambda ; t}$ is an eigenfunction of $\Delta_{\sigma, t}$ with eigenvalue $-\frac{(1-\sigma)^{2}}{t^{2}}-\lambda^{2}$. Moreover, $\phi_{\lambda, t}(0)=1$. For the second part let $f$ be a spherical function with eigenvalue $-\frac{(1-\sigma)^{2}}{t^{2}}-\lambda^{2}$ and consider

$$
f(x)=\left(1-\frac{\|x\|^{2}}{t^{2}}\right)^{\frac{1-\sigma+\mathrm{i} \lambda t}{2}} F\left(\frac{\|x\|^{2}}{t^{2}}\right)
$$

with $F$ a function defined on $\mathbb{B}_{t}^{n}$. Putting $\|x\|^{2}=r^{2}$ and writing $\Delta_{\sigma, t}$ in polar coordinates

$$
\Delta_{\sigma, t}=\frac{\left(1-\frac{r^{2}}{t^{2}}\right)^{2-\sigma}}{r^{n-1}} \frac{\partial}{\partial r}\left(r^{n-1}\left(1-\frac{r^{2}}{t^{2}}\right)^{\sigma} \frac{\partial}{\partial r}\right)+\frac{\sigma(2-n-\sigma)}{t^{2}}\left(1-\frac{r^{2}}{t^{2}}\right)+\frac{\left(1-\frac{r^{2}}{t^{2}}\right)^{2}}{r^{2}} \Delta_{\mathbb{S}^{n-1}}
$$

we see, by straightforward computations, that $F$ satisfies the following hypergeometric equation:

$$
\frac{r^{2}}{t^{2}}\left(1-\frac{r^{2}}{t^{2}}\right) F^{\prime \prime}\left(\frac{r^{2}}{t^{2}}\right)+\left(\frac{n}{2}-\left(\frac{n}{2}+\mathrm{i} \lambda t+1\right) t\right) F^{\prime}\left(\frac{r^{2}}{t^{2}}\right)-\frac{1+\mathrm{i} \lambda t}{2}\left(\frac{n-1+\mathrm{i} \lambda t}{2}\right) F\left(\frac{r^{2}}{t^{2}}\right)=0 .
$$

The smooth solutions at 0 of the last equation are multiples of ${ }_{2} F_{1}\left(\frac{1+\mathrm{i} \lambda t}{2}, \frac{n-1+\mathrm{i} \lambda t}{2} ; \frac{n}{2} ; \frac{\|x\|^{2}}{t^{2}}\right)$. Therefore, by (58) $f$ is a constant multiple of $\phi_{\lambda ; t}$.

Now we study the asymptotic behavior of $\phi_{\lambda ; t}$ at infinity. The resulting $c$-function is very important for the inversion of the $(\sigma, t)$-Helgason Fourier transform.

Lemma 2 For $\operatorname{Im}(\lambda)<0$ we have

$$
\lim _{s \rightarrow+\infty} \phi_{\lambda ; t}(t \tanh s) \mathrm{e}^{(-\mathrm{i} \lambda t+1-\sigma) s}=c(\lambda t)
$$

where $c(\lambda t)$ is the Harish-Chandra c-function given by

$$
c(\lambda t)=\frac{2^{1-\sigma-\mathrm{i} \lambda t} \Gamma(n / 2) \Gamma(\mathrm{i} \lambda t)}{\Gamma\left(\frac{n-1+\mathrm{i} \lambda t}{2}\right) \Gamma\left(\frac{1+\mathrm{i} \lambda t}{2}\right)} .
$$


Proof: Considering (59), (B.5) in Appendix B, (B.4) in Appendix B and the limit $\lim _{s \rightarrow+\infty} e^{s} / \cosh (s)=$ 2 we obtain

$$
\begin{aligned}
\lim _{s \rightarrow+\infty} \phi_{\lambda ; t}(t \tanh s) \mathrm{e}^{(-\mathrm{i} \lambda t+1-\sigma) s} & =\lim _{s \rightarrow \infty} \mathrm{e}^{(2-n-\sigma) s}(\cosh s)^{n-2+\sigma} \varphi_{\lambda t}^{\left(\frac{n}{2}-1, \frac{n}{2}-1\right)}(s) \mathrm{e}^{(-\mathrm{i} \lambda t+n-1) s} \\
& =2^{2-n-\sigma} c_{\frac{n}{2}-1, \frac{n}{2}-1}(\lambda t) \\
& =\frac{2^{1-\sigma-\mathrm{i} \lambda t} \Gamma(n / 2) \Gamma(\mathrm{i} \lambda t)}{\Gamma\left(\frac{n-1+\mathrm{i} \lambda t}{2}\right) \Gamma\left(\frac{1+\mathrm{i} \lambda t}{2}\right)}
\end{aligned}
$$

Finally, we prove the addition formula for the $(\sigma, t)$-spherical functions.

Proposition 8 For every $\lambda \in \mathbb{C}, t \in \mathbb{R}^{+}$, and $x, y \in \mathbb{B}_{t}^{n}$

$$
\begin{aligned}
\tau_{a} \phi_{\lambda ; t}(x) & =j_{a}(0) \int_{\mathbb{S}^{n-1}} e_{-\lambda, \xi ; t}(a) e_{\lambda, \xi ; t}(x) d \sigma(\xi) \\
& =j_{a}(0) \int_{\mathbb{S}^{n-1}} e_{\lambda, \xi ; t}(a) e_{-\lambda, \xi ; t}(x) d \sigma(\xi) .
\end{aligned}
$$

Proof: By (56) we have

$$
\begin{aligned}
\tau_{a} \phi_{\lambda ; t}(x) & =\int_{\mathbb{S}^{n-1}} \tau_{a} e_{\lambda, \xi ; t}(x) d \sigma(\xi) \\
& =j_{a}(0) \int_{\mathbb{S}^{n-1}} e_{\lambda, \xi ; t}(-a) e_{\lambda, a \oplus \xi ; t}(x) d \sigma(\xi) .
\end{aligned}
$$

Making the change of variables $a \oplus \xi=\xi^{\prime} \Leftrightarrow \xi=(-a) \oplus \xi^{\prime}$ the measure becomes

$$
d \sigma(\xi)=\left(\frac{1-\frac{\|a\|^{2}}{t^{2}}}{\left\|1+\frac{a \xi^{\prime}}{t}\right\|^{2}}\right)^{n-1} d \sigma\left(\xi^{\prime}\right)
$$

Therefore,

$$
\tau_{a} \phi_{\lambda ; t}(x)=j_{a}(0) \int_{\mathbb{S}^{n-1}} e_{\lambda,(-a) \oplus \xi^{\prime} ; t}(-a) e_{\lambda, \xi^{\prime} ; t}(x)\left(\frac{1-\frac{\|a\|^{2}}{t^{2}}}{\left\|1+\frac{a \xi^{\prime}}{t}\right\|^{2}}\right)^{n-1} d \sigma\left(\xi^{\prime}\right)
$$


Since

$$
\begin{aligned}
e_{\lambda,(-a) \oplus \xi^{\prime} ; t}(-a)\left(\frac{1-\frac{\|a\|^{2}}{t^{2}}}{\left\|1+\frac{a \xi^{\prime}}{t}\right\|^{2}}\right)^{n-1} & =\frac{\left(1-\frac{\|a\|^{2}}{t^{2}}\right)^{\frac{1-\sigma+\mathrm{i} \lambda t}{2}}}{\left\|\left(-\frac{a}{t}+\xi^{\prime}\right)\left(1+\frac{a \xi^{\prime}}{t}\right)^{-1}+\frac{a}{t}\right\|^{n-1+\mathrm{i} \lambda t}}\left(\frac{1-\frac{\|a\|^{2}}{t^{2}}}{\left\|1+\frac{a \xi^{\prime}}{t}\right\|^{2}}\right)^{n-1} \\
& =\frac{\left(1-\frac{\|a\|^{2}}{t^{2}}\right)^{\frac{1-\sigma+\mathrm{i} \lambda t}{2}}}{\left\|1+\frac{a \xi^{\prime}}{t}\right\|^{-(n-1+\mathrm{i} \lambda t)}\left(1-\frac{\|a\|^{2}}{t^{2}}\right)^{n-1+\mathrm{i} \lambda t}}\left(\frac{1-\frac{\|a\|^{2}}{t^{2}}}{\left\|1+\frac{a \xi^{\prime}}{t}\right\|^{2}}\right)^{n-1} \\
& =\frac{\left(1-\frac{\|a\|^{2}}{t^{2}}\right)^{\frac{1-\sigma-\mathrm{i} \lambda t}{2}}}{\left\|1+\frac{a \xi^{\prime}}{t}\right\|^{n-1-\mathrm{i} \lambda t}} \\
& =e_{-\lambda, \xi^{\prime} ; t}(a)\left(\left\|1+\frac{a \xi^{\prime}}{t}\right\|=\left\|\xi^{\prime}-\frac{a}{t}\right\|\right)
\end{aligned}
$$

we have

$$
\tau_{a} \phi_{\lambda ; t}(x)=j_{a}(0) \int_{\mathbb{S}^{n-1}} e_{-\lambda, \xi^{\prime} ; t}(a) e_{\lambda, \xi^{\prime} ; t}(x) d \sigma\left(\xi^{\prime}\right) .
$$

The second equality follows from the fact that $\phi_{\lambda ; t}$ is an even function of $\lambda$, i.e., $\phi_{\lambda ; t}=\phi_{-\lambda ; t}$.

\section{The $(\sigma, t)$-Helgason Fourier transform}

Definition 5 For $f \in C_{0}^{\infty}\left(\mathbb{B}_{t}^{n}\right), \lambda \in \mathbb{C}$ and $\xi \in \mathbb{S}^{n-1}$ we define the $(\sigma, t)$-Helgason Fourier transform of $f$ as

$$
\widehat{f}(\lambda, \xi ; t)=\int_{\mathbb{B}_{t}^{n}} f(x) e_{-\lambda, \xi ; t}(x) d \mu_{\sigma, t}(x) .
$$

Remark 3 If $f$ is a radial function i.e., $f(x)=f(\|x\|)$, then $\widehat{f}(\lambda, \xi ; t)$ is independent of $\xi$ and reduces by $(57)$ to the so called $(\sigma, t)$-spherical Fourier transform defined by

$$
\widehat{f}(\lambda ; t)=\int_{\mathbb{B}_{t}^{n}} f(x) \phi_{-\lambda ; t}(x) d \mu_{\sigma, t}(x) .
$$

Moreover, by (52) we recover in the Euclidean limit the Fourier transform in $\mathbb{R}^{n}$.

Since $\Delta_{\sigma, t}$ is a self-adjoint operator and by Proposition 5 we obtain the following result.

Proposition 9 If $f \in C_{0}^{\infty}\left(\mathbb{B}_{t}^{n}\right)$ then

$$
\widehat{\Delta_{\sigma, t} f}(\lambda, \xi ; t)=-\left(\frac{(1-\sigma)^{2}}{t^{2}}+\lambda^{2}\right) \widehat{f}(\lambda, \xi ; t) .
$$


Now we study the hyperbolic convolution theorem with respect to the $(\sigma, t)$-Helgason Fourier transform. We begin with the following lemma.

Lemma 3 For $a \in \mathbb{B}_{t}^{n}$ and $f \in C_{0}^{\infty}\left(\mathbb{B}_{t}^{n}\right)$

$$
\left.\widehat{\tau_{a} f}(\lambda, \xi ; t)=j_{a}(0) e_{-\lambda, \xi ; t}(a) \widehat{f}(\lambda,(-a) \oplus \xi) ; t\right) .
$$

Proof: By (39), (56) we have

$$
\begin{aligned}
\widehat{\tau_{a} f}(\lambda, \xi ; t) & =\int_{\mathbb{B}_{t}^{n}} \tau_{a} f(x) e_{-\lambda, \xi ; t}(x) d \mu_{\sigma, t}(x) \\
& =\int_{\mathbb{B}_{t}^{n}} f(x) \tau_{-a} e_{-\lambda, \xi ; t}(x) d \mu_{\sigma, t}(x) \\
& =j_{a}(0) e_{-\lambda, \xi ; t}(a) \int_{\mathbb{B}_{t}^{n}} f(x) e_{-\lambda,(-a) \oplus \xi ; t}(x) d \mu_{\sigma, t}(x) \\
& =j_{a}(0) e_{-\lambda, \xi ; t}(a) \hat{f}(\lambda,(-a) \oplus \xi ; t) .
\end{aligned}
$$

Theorem 5 (Generalised Hyperbolic Convolution Theorem) Let $f, g \in C_{0}^{\infty}\left(\mathbb{B}_{t}^{n}\right)$. Then

$$
\widehat{f * g}(\lambda, \xi)=\int_{\mathbb{B}_{t}^{n}} f(y) e_{-\lambda, \xi ; t}(y) \widehat{\widetilde{g}}_{y}(\lambda,(-y) \oplus \xi ; t) d \mu_{\sigma, t}(y)
$$

where $\widetilde{g}_{y}(x)=g(\operatorname{gyr}[y, x] x)$.

Proof: $\quad$ Let $I=\widehat{f * g}(\lambda, \xi)$. We have

$$
\begin{aligned}
I & =\int_{\mathbb{B}_{t}^{n}}\left(\int_{\mathbb{B}_{t}^{n}} f(y) \tau_{x} g(-y) j_{x}(x) d \mu_{\sigma, t}(y)\right) e_{-\lambda, \xi ; t}(x) d \mu_{\sigma, t}(x) \\
& =\int_{\mathbb{B}_{t}^{n}} f(y)\left(\int_{\mathbb{B}_{t}^{n}} \tau_{x} g(-y) e_{-\lambda, \xi ; t}(x) j_{x}(x) d \mu_{\sigma, t}(x)\right) d \mu_{\sigma, t}(y) \text { (Fubini) } \\
& =\int_{\mathbb{B}_{t}^{n}} f(y)\left(\int_{\mathbb{B}_{t}^{n}} \tau_{y} g(\operatorname{gyr}[x, y] x) e_{-\lambda, \xi ; t}(x) j_{y}(y) d \mu_{\sigma, t}(x)\right) d \mu_{\sigma, t}(y) \\
& =\int_{\mathbb{B}_{t}^{n}} f(y) \widehat{\tau_{y} \widetilde{g}_{y}}(\lambda, \xi ; t) j_{y}(y) d \mu_{\sigma, t}(y) \\
& \left.=\int_{\mathbb{B}_{t}^{n}} f(y) e_{-\lambda, \xi ; t}(y) \widehat{\widetilde{g}_{y}}(\lambda,(-y) \oplus \xi ; t) d \mu_{\sigma, t}(y) \quad \text { (by } \quad(66),(20)\right) .
\end{aligned}
$$

Corollary 5 Let $f, g \in C_{0}^{\infty}\left(\mathbb{B}_{t}^{n}\right)$ and $g$ radial. Then

$$
\widehat{f * g}(\lambda, \xi ; t)=\widehat{f}(\lambda, \xi ; t) \widehat{g}(\lambda ; t) .
$$


Since in the limit $t \rightarrow+\infty$ gyrations reduce to the identity and $(-y) \oplus \xi$ reduces to $\xi$, formula $(67)$ converges in the Euclidean limit to the well-know convolution Theorem: $\widehat{f * g}=\widehat{f} \cdot \widehat{g}$.

Next proposition shows that the Fourier coefficients of a given function $f \in C_{0}^{\infty}\left(\mathbb{B}_{t}^{n}\right)$ can be related with the $(\sigma, t)$-convolution.

Proposition 10 For $f \in C_{0}^{\infty}\left(\mathbb{B}_{t}^{n}\right)$ and $\lambda \in \mathbb{C}$,

$$
f * \phi_{\lambda ; t}(x)=\int_{\mathbb{S}^{n-1}} \widehat{f}(\lambda, \xi ; t) e_{\lambda, \xi ; t}(x) d \sigma(\xi) .
$$

Proof: By (62), (20), Fubini's Theorem, and the fact that $\phi_{\lambda, t}$ is a radial function we have

$$
\begin{aligned}
f * \phi_{\lambda ; t}(x) & =\int_{\mathbb{B}_{t}^{n}} f(y) \tau_{x} \phi_{\lambda ; t}(y) j_{x}(x) d \mu_{\sigma, t}(y) \\
& =\int_{\mathbb{B}_{t}^{n}} f(y)\left(\int_{\mathbb{S}^{n-1}} e_{\lambda, \xi ; t}(x) e_{-\lambda, \xi ; t}(y) j_{x}(0) j_{x}(x) d \sigma(\xi)\right) d \mu_{\sigma, t}(y) \\
& =\int_{\mathbb{S}^{n-1}}\left(\int_{\mathbb{B}_{t}^{n}} f(y) e_{-\lambda, \xi ; t}(y) d \mu_{\sigma, t}(y)\right) e_{\lambda, \xi ; t}(x) d \sigma(\xi) \\
& =\int_{\mathbb{S}^{n-1}} \widehat{f}(\lambda, \xi ; t) e_{\lambda, \xi ; t}(x) d \sigma(\xi) .
\end{aligned}
$$

\section{Inversion of the $(\sigma, t)$-Helgason Fourier transform and Plancherel's Theorem}

We obtain first an inversion formula for the radial case, that is, for the $(\sigma, t)$-spherical Fourier transform and then we will derive a general inversion formula for the $(\sigma, t)$-Helgason Fourier transform.

Lemma 4 The $(\sigma, t)$-spherical Fourier transform $\mathcal{H}$ can be written as

$$
\mathcal{H}=\mathcal{J}_{\frac{n}{2}-1, \frac{n}{2}-1} \circ M_{\sigma}
$$

where $\mathcal{J}_{\frac{n}{2}-1, \frac{n}{2}-1}$ is the Jacobi transform (B.1 in Appendix B) with parameters $\alpha=\beta=\frac{n}{2}-1$ and

$$
\left(M_{\sigma} f\right)(s):=2^{2-2 n} A_{n-1} t^{n}(\cosh s)^{2-n-\sigma} f(t \tanh s) .
$$

Proof: Integrating (64) in polar coordinates $x=r \xi$ and making the change of variables $r=t \tanh s$ we obtain

$$
\begin{aligned}
\widehat{f}(\lambda ; t) & =A_{n-1} \int_{0}^{t} f(r) \phi_{-\lambda ; t}(r)\left(1-\frac{r^{2}}{t^{2}}\right)^{\sigma-2} r^{n-1} d r \\
& =A_{n-1} \int_{0}^{+\infty} f(t \tanh s) \phi_{-\lambda ; t}(t \tanh s)(\cosh s)^{-2 \sigma+4-2}(\tanh s)^{n-1} t^{n} d s
\end{aligned}
$$


Applying (59) yields

$$
\begin{aligned}
\widehat{f}(\lambda ; t) & =A_{n-1} \int_{0}^{+\infty} f(t \tanh s) \varphi_{\lambda t}^{\left(\frac{n}{2}-1, \frac{n}{2}-1\right)}(s)(\sinh s)^{n-1}(\cosh s)^{1-\sigma} t^{n} d s \\
& =2^{2-2 n} A_{n-1} \int_{0}^{+\infty} t^{n}(\cosh s)^{2-\sigma-n} f(t \tanh s) \varphi_{\lambda t}^{\left(\frac{n}{2}-1, \frac{n}{2}-1\right)}(s)(2 \sinh s)^{n-1}(2 \cosh s)^{n-1} d s \\
& =\int_{0}^{+\infty}\left(M_{\sigma} f\right)(s) \varphi_{\lambda t}^{\left(\frac{n}{2}-1, \frac{n}{2}-1\right)}(s)(2 \sinh s)^{n-1}(2 \cosh s)^{n-1} d s \\
& =\left(\mathcal{J}_{\frac{n}{2}-1, \frac{n}{2}-1} \circ M_{\sigma} f\right)(\lambda t) .
\end{aligned}
$$

In the sequel $C_{0, R}^{\infty}\left(\mathbb{B}_{t}^{n}\right)$ denotes the space of all radial $C^{\infty}$ functions on $\mathbb{B}_{t}^{n}$ with compact support and $C_{n, t, \sigma}=\frac{1}{2^{-1+2 \sigma} t^{n-1} \pi A_{n-1}}$.

Theorem 6 For all $f \in C_{0, R}^{\infty}\left(\mathbb{B}_{t}^{n}\right)$ we have the inversion formula

$$
f(x)=C_{n, t, \sigma} \int_{0}^{+\infty} \widehat{f}(\lambda ; t) \phi_{\lambda ; t}(x)|c(\lambda t)|^{-2} t d \lambda .
$$

Proof: Applying the formula (B.3) (in Appendix B) for the Jacobi transform and Lemma 4 we obtain

$$
\begin{aligned}
M_{\sigma} f(s) & =\frac{1}{2 \pi} \int_{0}^{+\infty} \widehat{f}(\lambda ; t) \varphi_{\lambda t}^{\left(\frac{n}{2}-1, \frac{n}{2}-1\right)}(s)\left|c_{\frac{n}{2}-1, \frac{n}{2}-1}(\lambda t)\right|^{-2} t d \lambda \\
& =\frac{1}{2 \pi} \int_{0}^{+\infty} \widehat{f}(\lambda ; t)(\cosh s)^{2-n-\sigma} \phi_{\lambda ; t}(x) \frac{|c(\lambda t)|^{-2}}{2^{-4+2 n+2 \sigma}} t d \lambda .
\end{aligned}
$$

In the last equality we use (59) and (61). By the definition (70) of $M_{\sigma} f$ we obtain

$$
f(t \tanh s)=C_{n, t, \sigma} \int_{0}^{+\infty} \widehat{f}(\lambda ; t) \phi_{\lambda ; t}(x)|c(\lambda t)|^{-2} d \lambda
$$

Since $t \tanh s=r$ we obtain the desired result.

Remark 4 The inversion formula (71) can be written as

$$
f(x)=\frac{C_{n, t, \sigma}}{2} \int_{\mathbb{R}} \widehat{f}(\lambda ; t) \phi_{\lambda ; t}(x)|c(\lambda t)|^{-2} d \lambda
$$

since the integrand is an even function of $\lambda \in \mathbb{R}$. Note that $f$ is radial and therefore $\widehat{f}(\lambda ; t)$ is an even function of $\lambda, \phi_{\lambda ; t}=\phi_{-\lambda ; t}$, and $|c(-\lambda t)|=|\overline{c(\lambda t)}|=|c(\lambda t)|$, for $\lambda \in \mathbb{R}$.

Finally, we state our main results, the inversion formula for the $(\sigma, t)$-Helgason Fourier transform and the Plancherel's Theorem. 
Theorem 7 If $f \in C_{0}^{\infty}\left(\mathbb{B}_{t}^{n}\right)$ then

$$
f(x)=C_{n, t, \sigma} \int_{0}^{+\infty} \int_{\mathbb{S}^{n-1}} \widehat{f}(\lambda, \xi ; t) e_{\lambda, \xi ; t}(x)|c(\lambda t)|^{-2} d \sigma(\xi) d \lambda .
$$

Proof: Given $f \in C_{0}^{\infty}\left(B_{n}^{t}\right)$ and $x, y \in \mathbb{B}_{t}^{n}$ we consider the radial function

$$
f_{x}(y)=\int_{\mathrm{SO}(n)} \tau_{K^{-1} x} f(-K y) j_{x}(x) d K,
$$

where $K \in \mathrm{SO}(n)$ and $d K$ is the normalised Haar measure on $\mathrm{SO}(n)$. Applying the inversion formula (71) we get

$$
f_{x}(y)=C_{n, t, \sigma} \int_{0}^{+\infty} \widehat{f}_{x}(\lambda ; t) \phi_{\lambda ; t}(y)|c(\lambda t)|^{-2} d \lambda .
$$

By (64), Fubini's Theorem, and the change of variables $K y \mapsto z$ we have

$$
\begin{aligned}
\widehat{f}_{x}(\lambda ; t) & =\int_{\mathbb{B}_{t}^{n}}\left(\int_{\mathrm{SO}(n)} \tau_{K^{-1} x} f(-K y) j_{x}(x) d K\right) \phi_{-\lambda ; t}(y) d \mu_{\sigma, t}(y) \\
& =\int_{\mathrm{SO}(n)}\left(\int_{\mathbb{B}_{t}^{n}} f(x \oplus(-K y)) j_{K^{-1} x}(y) j_{x}(x) \phi_{-\lambda ; t}(y) d \mu_{\sigma, t}(y)\right) d K \\
& =\int_{\mathrm{SO}(n)}\left(\int_{\mathbb{B}_{t}^{n}} f(x \oplus(-z)) j_{x}(z) j_{x}(x) \phi_{-\lambda ; t}(z) d \mu_{\sigma, t}(z)\right) d K \\
& =\int_{\mathbb{B}_{t}^{n}} \tau_{x} f(-z) \phi_{-\lambda ; t}(z) j_{x}(x) d \mu_{\sigma, t}(z) \\
& =\left(f * \phi_{\lambda ; t}\right)(x) .
\end{aligned}
$$

Since $f(x)=f_{x}(0)$ it follows from $(74),(75)$, and (69) that

$$
\begin{aligned}
f(x) & =C_{n, t, \sigma} \int_{0}^{+\infty} \widehat{f}_{x}(\lambda ; t) \phi_{\lambda ; t}(0)|c(\lambda t)|^{-2} d \lambda \\
& =C_{n, t, \sigma} \int_{0}^{+\infty}\left(f * \phi_{\lambda ; t}\right)(x)|c(\lambda t)|^{-2} d \lambda \\
& =C_{n, t, \sigma} \int_{0}^{+\infty} \int_{\mathbb{S}^{n-1}} \widehat{f}(\lambda, \xi ; t) e_{\lambda, \xi ; t}(x)|c(\lambda t)|^{-2} d \sigma(\xi) d \lambda .
\end{aligned}
$$

Remark 5 Applying the inversion formula (72) in the proof of Theorem 7 we can write the inversion formula (73) as

$$
f(x)=\frac{C_{n, t, \sigma}}{2} \int_{\mathbb{R}} \int_{\mathbb{S}^{n-1}} \widehat{f}(\lambda, \xi ; t) e_{\lambda, \xi ; t}(x)|c(\lambda t)|^{-2} d \sigma(\xi) d \lambda .
$$

Theorem 8 (Plancherel's Theorem) The $(\sigma, t)$-Helgason Fourier transform extends to an isometry from $L^{2}\left(\mathbb{B}_{t}^{n}, d \mu_{\sigma, t}\right)$ onto $L^{2}\left(\mathbb{R}^{+} \times \mathbb{S}^{n-1}, C_{n, t, \sigma}|c(\lambda t)|^{-2} d \lambda d \sigma\right)$, i.e.,

$$
\int_{\mathbb{B}_{t}^{n}}|f(x)|^{2} d \mu_{\sigma, t}(x)=C_{n, t, \sigma} \int_{0}^{+\infty} \int_{\mathbb{S}^{n-1}}|\widehat{f}(\lambda, \xi ; t)|^{2}|c(\lambda t)|^{-2} d \sigma(\xi) d \lambda .
$$


Proof: For $f, g \in C_{0}^{\infty}\left(\mathbb{B}_{t}^{n}\right)$ we obtain Parseval's relation by the inversion formula (73) and Fubini's Theorem:

$$
\begin{aligned}
& C_{n, t, \sigma} \int_{0}^{+\infty} \int_{S^{n-1}} \widehat{f}(\lambda, \xi ; t) \overline{\widehat{g}(\lambda, \xi ; t)}|c(\lambda t)|^{-2} d \sigma(\xi) d \lambda \\
& =C_{n, t, \sigma} \int_{0}^{+\infty} \int_{S^{n-1}} \widehat{f}(\lambda, \xi ; t) \int_{\mathbb{B}_{t}^{n}} \overline{g(x)} e_{\lambda, \xi ; t}(x) d \mu_{\sigma, t}(x)|c(\lambda t)|^{-2} d \sigma(\xi) d \lambda \\
& =\int_{\mathbb{B}_{t}^{n}}\left[C_{n, t, \sigma} \int_{0}^{+\infty} \int_{S^{n-1}} \widehat{f}(\lambda, \xi ; t) e_{\lambda, \xi ; t}(x)|c(\lambda t)|^{-2} d \sigma(\xi) d \lambda\right] \overline{g(x)} d \mu_{\sigma, t}(x) \\
& =\int_{\mathbb{B}_{t}^{n}} f(x) \overline{g(x)} d \mu_{\sigma, t}(x) .
\end{aligned}
$$

By taking $f=g$ we obtain $(77)$ for $f \in C_{0}^{\infty}\left(\mathbb{B}_{t}^{n}\right)$ and the result can be extended to $L^{2}\left(\mathbb{B}_{t}^{n}, d \mu_{\sigma, t}\right)$ since $C_{0}^{\infty}\left(\mathbb{B}_{t}^{n}\right)$ is dense in $L^{2}\left(\mathbb{B}_{t}^{n}, d \mu_{\sigma, t}\right)$. It remains to prove the surjectivity of the $(\sigma, t)$-Helgason Fourier transform. This can be done in a similar way as in ([17], Theorem 6.14) and therefore we omit the details.

Having obtained the main results we now study the limit $t \rightarrow+\infty$ of the previous results. It is anticipated that in the Euclidean limit we recover the usual inversion formula for the Fourier transform and Parseval's Theorem on $\mathbb{R}^{n}$. To see that this is indeed the case, we begin by noting that from the relation

$$
\Gamma(z) \Gamma\left(z+\frac{1}{2}\right)=2^{1-2 z} \sqrt{\pi} \Gamma(2 z)
$$

the $c$-function (60) simplifies to

$$
c(\lambda t)=\frac{2^{-\sigma}}{\sqrt{\pi}} \Gamma\left(\frac{n}{2}\right) \frac{\Gamma\left(\frac{\mathrm{i} \lambda t}{2}\right)}{\Gamma\left(\frac{n-1+\mathrm{i} \lambda t}{2}\right)} .
$$

Therefore, we have

$$
\frac{1}{|c(\lambda t)|^{2}}=\frac{2^{2 \sigma} \pi}{\left(\Gamma\left(\frac{n}{2}\right)\right)^{2}}\left|\frac{\Gamma\left(\frac{n-1}{2}+\frac{\mathrm{i} \lambda t}{2}\right)}{\Gamma\left(\frac{\mathrm{i} \lambda t}{2}\right)}\right|^{2}=\frac{4^{\sigma-1}\left(A_{n-1}\right)^{2}}{\pi^{n-1}}\left|\frac{\Gamma\left(\frac{n-1}{2}+\frac{\mathrm{i} \lambda t}{2}\right)}{\Gamma\left(\frac{\mathrm{i} \lambda t}{2}\right)}\right|^{2},
$$

with $A_{n-1}=\frac{2 \pi^{\frac{n}{2}}}{\Gamma\left(\frac{n}{2}\right)}$ being the surface area of $\mathbb{S}^{n-1}$. Finally, using $(78)$ the $(\sigma, t)$-Helgason inverse Fourier transform (73) simplifies to

$$
\begin{aligned}
f(x) & =\frac{A_{n-1}}{2 \pi^{n} t^{n-1}} \int_{0}^{+\infty} \int_{\mathbb{S}^{n-1}} \widehat{f}(\lambda, \xi ; t) e_{\lambda, \xi ; t}(x)\left|\frac{\Gamma\left(\frac{n-1}{2}+\frac{\mathrm{i} \lambda t}{2}\right)}{\Gamma\left(\frac{\mathrm{i} \lambda t}{2}\right)}\right|^{2} \frac{1}{\left(\frac{\lambda t}{2}\right)^{n-1}}\left(\frac{\lambda t}{2}\right)^{n-1} d \sigma(\xi) d \lambda \\
& =\frac{1}{(2 \pi)^{n}} \int_{0}^{+\infty} \int_{\mathbb{S}^{n-1}} \widehat{f}(\lambda, \xi ; t) e_{\lambda, \xi ; t}(x) \frac{\lambda^{n-1}}{N^{(n)}(\lambda t)} d \xi d \lambda
\end{aligned}
$$

with $N^{(n)}(\lambda t)$ given by (55). Some particular values are $N^{(1)}(\lambda t)=1, N^{(2)}(\lambda t)=\operatorname{coth}\left(\frac{\lambda t}{2}\right), N^{(3)}=1$, and $N^{(4)}(\lambda t)=\frac{(\lambda t)^{2} \operatorname{coth}\left(\frac{\pi \lambda t}{2}\right)}{1+(\lambda t)^{2}}$. Since $\lim _{t \rightarrow+\infty} N^{(n)}(\lambda t)=1$, for any $n \in \mathbb{N}$ and $\lambda \in \mathbb{R}^{+}$(see [3]), we see 
that in the Euclidean limit the $(\sigma, t)$-Helgason inverse Fourier transform (79) converges to the usual inverse Fourier transform in $\mathbb{R}^{n}$ written in polar coordinates:

$$
f(x)=\frac{1}{(2 \pi)^{n}} \int_{0}^{+\infty} \int_{\mathbb{S}^{n-1}} \widehat{f}(\lambda \xi) \mathrm{e}^{\mathrm{i}\langle x, \lambda \xi\rangle} \lambda^{n-1} d \xi d \lambda, \quad x, \lambda \xi \in \mathbb{R}^{n} .
$$

Finally, Plancherel's Theorem (77) can be written as

$$
\int_{\mathbb{B}_{t}^{n}}|f(x)|^{2} d \mu_{\sigma, t}(x)=\frac{1}{(2 \pi)^{n}} \int_{0}^{+\infty} \int_{\mathbb{S}^{n-1}}|\widehat{f}(\lambda, \xi ; t)|^{2} \frac{\lambda^{n-1}}{N^{(n)}(\lambda t)} d \xi d \lambda
$$

and, therefore, we have an isometry between the spaces $L^{2}\left(\mathbb{B}_{t}^{n}, d \mu_{\sigma, t}\right)$ and $L^{2}\left(\mathbb{R}^{+} \times \mathbb{S}^{n-1}, \frac{\lambda^{n-1}}{(2 \pi)^{n} N^{(n)}(\lambda t)} d \lambda d \xi\right)$. Applying the limit $t \rightarrow+\infty$ to (80) we recover Plancherel's Theorem in $\mathbb{R}^{n}$ :

$$
\int_{\mathbb{R}^{n}}|f(x)|^{2} d x=\frac{1}{(2 \pi)^{n}} \int_{0}^{+\infty} \int_{\mathbb{S}^{n-1}}|\widehat{f}(\lambda \xi)|^{2} \lambda^{n-1} d \xi d \lambda
$$

\section{Diffusive wavelets on the ball}

The idea of diffusive wavelets is to construct wavelets by a diffusion process. For an overview of diffusive wavelets on groups and homogeneous spaces we refer to $[6,7]$.

Definition 6 (Diffusive wavelets on a Lie group) Let $p_{\tau}$ be a diffusive approximate identity and $\alpha(\rho)>$ 0 a given weight function. A family $\psi_{\rho} \in L^{2}(G)$ is called diffusive wavelet family if it satisfies the admissibility condition

$$
p_{\tau}=\int_{0}^{\infty} \breve{\psi}_{\rho} * \psi_{\rho} \alpha(\rho) d \rho
$$

where $\breve{\psi}_{\rho}(g)=\overline{\psi_{\rho}\left(g^{-1}\right)}$.

We want to construct a diffusive wavelet family from the heat kernel on $\mathbb{B}_{t}^{n}$. Let's consider the heat equation associated to $\Delta_{\sigma, t}$ :

$$
\left\{\begin{array}{l}
\partial_{\tau} u(x, \tau)=\Delta_{\sigma, t} u(x, \tau) \\
u(x, 0)=f(x)
\end{array},(x, \tau) \in \mathbb{B}_{t}^{n} \times \mathbb{R}^{+}\right.
$$

where $f \in C_{0}^{\infty}\left(\mathbb{B}_{t}^{n}\right), \tau>0$ is the time evolution parameter and $u$ is assumed to be a $C^{\infty}$ function and compactly supported in the spatial variable. Using the $(\sigma, t)$-Helgason Fourier transform in the spatial variable and by (65) we obtain

$$
\widehat{u}(\lambda, \xi, \tau ; t)=\mathrm{e}^{-\left(\frac{(1-\sigma)^{2}}{t^{2}}+\lambda^{2}\right) \tau} \widehat{f}(\lambda, \xi ; t) .
$$

Therefore, by (68) and applying the inverse $(\sigma, t)$-Helgason Fourier transform, the solution of the heat equation is given as convolution $u(x, \tau ; t)=\left(p_{\tau, t} * f\right)(x)$, where the heat kernel $p_{\tau, t}$ is given by

$$
\widehat{p_{\tau, t}}(\lambda)=\mathrm{e}^{-\left(\frac{(1-\sigma)^{2}}{t^{2}}+\lambda^{2}\right) \tau} \quad \text { and } \quad p_{\tau, t}(x)=\mathcal{F}_{H}^{-1}\left[\mathrm{e}^{-\left(\frac{(1-\sigma)^{2}}{t^{2}}+\lambda^{2}\right) \tau}\right]
$$


with $\mathcal{F}_{H}^{-1}[\cdot]$ being the inverse of the $(\sigma, t)$-Helgason Fourier transform. A closed formula for the heat kernel in the unit ball was obtained in [17, Thm. 7.3].

Given a weight function $\alpha(\rho)>0$ the heat wavelet family on $\mathbb{B}_{t}^{n}$ is given on the Fourier side by (see [6])

$$
\widehat{\psi}_{\rho, t}(\lambda)=\frac{1}{\sqrt{\alpha(\rho)}} \sqrt{\frac{\left(1-\sigma^{2}\right)}{t^{2}}+\lambda^{2}} \mathrm{e}^{-\left(\frac{\left(1-\sigma^{2}\right)}{t^{2}}+\lambda^{2}\right) \frac{\rho}{2}} .
$$

In the limit $t \rightarrow+\infty$ this wavelet family coincide with the classical heat wavelet family in $\mathbb{R}^{n}$. For $f \in L^{2}\left(\mathbb{B}_{t}^{n}\right)$ we can define a wavelet transform on the ball by

$$
W_{\psi} f(\rho, b ; t)=\left(f * \breve{\psi}_{\rho, t}\right)(b)=\int_{\mathbb{B}_{t}^{n}} f(x) \tau_{b} \breve{\psi}_{\rho, t}(-x) j_{b}(b) d \mu_{\sigma, t}(x)
$$

where $\breve{\psi}_{\rho, t}(x)=\overline{\psi_{\rho, t}(-x)}$.

The wavelet transform $W: L^{2}\left(\mathbb{B}_{t}^{n}, d \mu_{\sigma, t}\right) \rightarrow L^{2}\left(\mathbb{R}_{+} \times \mathbb{B}_{t}^{n}, \alpha(\rho) d \rho d \mu_{\sigma, t}(b)\right)$ is a unitary operator and it can be inverted on its range by

$$
f(x)=\int_{0}^{+\infty}\left(W_{\psi} f\right)(\rho, \cdot, t) * \psi_{\rho, t} \alpha(\rho) d \rho \quad \forall f \in L^{2}\left(\mathbb{B}_{t}^{n}\right) .
$$

\section{Appendices}

\section{A Spherical harmonics}

A spherical harmonic of degree $k \geq 0$ denoted by $Y_{k}$ is the restriction to $\mathbb{S}^{n-1}$ of a homogeneous harmonic polynomial in $\mathbb{R}^{n}$. The set of all spherical harmonics of degree $k$ is denoted by $\mathcal{H}_{k}\left(\mathbb{S}^{n-1}\right)$. This space is a finite dimensional subspace of $L^{2}\left(\mathbb{S}^{n-1}\right)$ and we have the direct sum decomposition

$$
L^{2}\left(\mathbb{S}^{n-1}\right)=\bigoplus_{k=0}^{\infty} \mathcal{H}_{k}\left(\mathbb{S}^{n-1}\right)
$$

The following integrals are obtained from the generalisation of Lemma 2.4 in [17].

Lemma 5 [17] Let $\nu \in \mathbb{C}, k \in \mathbb{N}_{0}, t \in \mathbb{R}^{+}$, and $Y_{k} \in \mathcal{H}_{k}\left(\mathbb{S}^{n-1}\right)$. Then

$$
\int_{\mathbb{S}^{n-1}} \frac{Y_{k}(\xi)}{\left\|\frac{x}{t}-\xi\right\|^{2 \nu}} d \sigma(\xi)=\frac{(\nu)_{k}}{(n / 2)_{k}}{ }_{2} F_{1}\left(k+\nu, \nu-\frac{n}{2}+1 ; k+\frac{n}{2} ; \frac{\|x\|^{2}}{t^{2}}\right) \frac{\|x\|^{k}}{t^{k}} Y_{k}\left(x^{\prime}\right)
$$

where $x \in \mathbb{B}_{t}^{n}, x^{\prime}=\|x\|^{-1} x,(\nu)_{k}$ denotes the Pochammer symbol, and $d \sigma$ is the normalised surface measure on $\mathbb{S}^{n-1}$. In particular, when $k=0$, we have

$$
\int_{\mathbb{S}^{n-1}} \frac{1}{\left\|\frac{x}{t}-\xi\right\|^{2 \nu}} d \sigma(\xi)={ }_{2} F_{1}\left(\nu, \nu-\frac{n}{2}+1 ; \frac{n}{2} ; \frac{\|x\|^{2}}{t^{2}}\right) .
$$


The Gauss Hypergeometric function ${ }_{2} F_{1}$ is an analytic function for $|z|<1$ defined by

$$
{ }_{2} F_{1}(a, b ; c ; z)=\sum_{k=0}^{\infty} \frac{(a)_{k}(b)_{k}}{(c)_{k}} \frac{z^{k}}{k !}
$$

with $c \notin-\mathbb{N}_{0}$. If $\operatorname{Re}(c-a-b)>0$ and $c \notin-\mathbb{N}_{0}$ then exists the limit $\lim _{t \rightarrow 1^{-}} F_{1}(a, b ; c ; t)$ and equals

$$
{ }_{2} F_{1}(a, b ; c ; 1)=\frac{\Gamma(c) \Gamma(c-a-b)}{\Gamma(c-a) \Gamma(c-b)} .
$$

Some useful properties of this function are

$$
\begin{gathered}
{ }_{2} F_{1}(a, b ; c ; z)=(1-z)^{c-a-b}{ }_{2} F_{1}(c-a, c-b ; c ; z) \\
{ }_{2} F_{1}(a, b ; c ; z)=(1-z)^{-a}{ }_{2} F_{1}\left(a, c-b ; c ; \frac{z}{z-1}\right) \\
\frac{d}{d z}{ }_{2} F_{1}(a, b ; c ; z)=\frac{a b}{c}{ }_{2} F_{1}(a+1, b+1 ; c+1 ; z) .
\end{gathered}
$$

\section{B Jacobi functions}

The classical theory of Jacobi functions involves the parameters $\alpha, \beta, \lambda \in \mathbb{C}$ (see [14, 15]). Here we introduce the additional parameter $t \in \mathbb{R}^{+}$since we develop our hyperbolic harmonic analysis on a ball of arbitrary radius $t$. For $\alpha, \beta, \lambda \in \mathbb{C}, t \in \mathbb{R}^{+}$, and $\alpha \neq-1,-2, \ldots$, we define the Jacobi transform as

$$
\mathcal{J}_{\alpha, \beta} g(\lambda t)=\int_{0}^{+\infty} g(r) \varphi_{\lambda t}^{(\alpha, \beta)}(r) \omega_{\alpha, \beta}(r) d r
$$

for all functions $g$ defined on $\mathbb{R}^{+}$for which the integral (B.1) is well defined. The weight function $\omega_{\alpha, \beta}$ is given by

$$
\omega_{\alpha, \beta}(r)=(2 \sinh (r))^{2 \alpha+1}(2 \cosh (r))^{2 \beta+1}
$$

and the function $\varphi_{\lambda t}^{(\alpha, \beta)}(r)$ denotes the Jacobi function which is defined as the even $C^{\infty}$ function on $\mathbb{R}$ that equals 1 at 0 and satisfies the Jacobi differential equation

$$
\left(\frac{d^{2}}{d r^{2}}+((2 \alpha+1) \operatorname{coth}(r)+(2 \beta+1) \tanh (r)) \frac{d}{d r}+(\lambda t)^{2}+(\alpha+\beta+1)^{2}\right) \varphi_{\lambda t}^{(\alpha, \beta)}(r)=0 .
$$

The function $\varphi_{\lambda t}^{(\alpha, \beta)}(r)$ can be expressed as an hypergeometric function

$$
\varphi_{\lambda t}^{(\alpha, \beta)}(r)={ }_{2} F_{1}\left(\frac{\alpha+\beta+1+\mathrm{i} \lambda t}{2}, \frac{\alpha+\beta+1-\mathrm{i} \lambda t}{2} ; \alpha+1 ;-\sinh ^{2}(r)\right) .
$$

Since $\varphi_{\lambda t}^{(\alpha, \beta)}$ are even functions of $\lambda t \in \mathbb{C}$ then $\mathcal{J}_{\alpha, \beta} g(\lambda t)$ is an even function of $\lambda t$. Payley-Wiener Theorem and some inversion formulas for the Jacobi transform are found in [15]. We denote by $C_{0, R}^{\infty}(\mathbb{R})$ the space of even $C^{\infty}$-functions with compact support on $\mathbb{R}$ and $\mathcal{E}$ the space of even and entire functions $g$ for which there are positive constants $A_{g}$ and $C_{g, n}, n=0,1,2, \ldots$, such that for all $\lambda \in \mathbb{C}$ and all $n=0,1,2, \ldots$

$$
|g(x)| \leq C_{g, n}(1+|\lambda|)^{-n} e^{A_{g}|\operatorname{Im}(\lambda)|}
$$

where $\operatorname{Im}(\lambda)$ denotes the imaginary part of $\lambda$. 
Theorem 9 ([15],p.8) (Payley-Wiener Theorem) For all $\alpha, \beta \in \mathbb{C}$ with $\alpha \neq-1,-2, \ldots$ the Jacobi transform is bijective from $C_{0, R}^{\infty}(\mathbb{R})$ onto $\mathcal{E}$.

The Jacobi transform can be inverted under some conditions [15]. Here we only refer to the case which is used in this paper.

Theorem 10 ([15],p.9) Let $\alpha, \beta \in \mathbb{R}$ such that $\alpha>-1, \alpha \pm \beta+1 \geq 0$. Then for every $g \in C_{0, R}^{\infty}(\mathbb{R})$ we have

$$
g(r)=\frac{1}{2 \pi} \int_{0}^{+\infty}\left(\mathcal{J}_{\alpha, \beta} g\right)(\lambda t) \varphi_{\lambda t}^{(\alpha, \beta)}(r)\left|c_{\alpha, \beta}(\lambda t)\right|^{-2} t d \lambda,
$$

where $c_{\alpha, \beta}(\lambda t)$ is the Harish-Chandra c-function associated to $\mathcal{J}_{\alpha, \beta}(\lambda t)$ given by

$$
c_{\alpha, \beta}(\lambda t)=\frac{2^{\alpha+\beta+1-\mathrm{i} \lambda t} \Gamma(\alpha+1) \Gamma(\mathrm{i} \lambda t)}{\Gamma\left(\frac{\alpha+\beta+1+\mathrm{i} \lambda t}{2}\right) \Gamma\left(\frac{\alpha-\beta+1+\mathrm{i} \lambda t}{2}\right)} .
$$

This theorem provides a generalisation of Theorem 2.3 in [15] for arbitrary $t \in \mathbb{R}^{+}$. From [15] and considering $t \in \mathbb{R}^{+}$arbitrary we have the following asymptotic behavior of $\phi_{\lambda t}^{\alpha, \beta}$ for $\operatorname{Im}(\lambda)<0$ :

$$
\lim _{r \rightarrow+\infty} \varphi_{\lambda t}^{(\alpha, \beta)}(r) e^{(-\mathrm{i} \lambda t+\alpha+\beta+1) r}=c_{\alpha, \beta}(\lambda t) .
$$

\section{Acknowledgements}

This work was supported by Portuguese funds through the CIDMA - Center for Research and Development in Mathematics and Applications, and the Portuguese Foundation for Science and Technology ("FCT - Fundação para a Ciência e a Tecnologia"), within project PEst-OE/MAT/UI4106/2014.

\section{References}

[1] Ahlfors, L., Möbius transformations in several dimensions, University of Minnesota School of Mathematics, Minneapolis, 1981.

[2] Ahlfors, L., Möbius transformations in $\mathbb{R}^{n}$ expressed through $2 \times 2$ matrices of Clifford numbers, Complex Variables 5 (1986), 215-221.

[3] Alonso, M., Pogosyan, G., Wolf, K., Wigner functions for curved spaces I: On hyperboloids, J. Math. Phys. $7(12)$ (2002), 5857-5871.

[4] Cnops, J., Hurwitz pairs and applications of Möbius transformations, Habilitation dissertation, Universiteit Gent, Faculteit van de Wetenschappen, 1994.

[5] Delanghe, R., Sommen, F., Souček, V., Clifford algebras and spinor-valued functions, Kluwer Acad. Publ., Dordrecht, 1992.

[6] Ebert, S., Wavelets and Lie groups and homogeneous spaces, PhD thesis, TU Bergakademie Freiberg, 2011.

[7] Ebert, S., Wirth, J., Diffusive wavelets on groups and homogeneous spaces, Proc. Roy. Soc. Edinburgh 141A:497-520, 2011,

[8] Ferreira, M., Factorizations of Möbius gyrogroups, Adv. Appl. Clifford Algebr. 19(2) (2009), 303-323. 
[9] Ferreira, M., Ren, G., Möbius gyrogroups: a Clifford algebra approach, J. Algebra 328(1) (2011), 230-253.

[10] Foguel, T., Ungar, A. A., Gyrogroups and the decomposition of groups into twisted subgroups and subgroups, Pac. J. Math, 197(1) (2001), 1-11.

[11] Helgason, S., Groups and Geometric Analysis, Academic Press, Orlando, FL, 1984.

[12] Helgason, S., Geometric Analysis on Symmetric Spaces, AMS, Providence, RI, 1994.

[13] Hua, L. K., Starting with the unit circle, Springer, New-York, 1981.

[14] Koornwinder, T. H., A new proof of a Payley-Wiener type theorem for the Jacobi transform, Ark. Mat. 13 (1975), 145-159.

[15] Koornwinder, T. H., Jacobi functions and analysis on non-compact semisimple groups, Special Functions Group Theoretical Aspects and Applications, Reidel, Dordrecht (1984), 1-84.

[16] Lal, R, Yadav, A., Topological Right Gyrogroups and Gyrotransversals, Comm. Algebra 41(9) (2013), 35593575 .

[17] Liu, C., Peng, L., Generalized Helgason-Fourier transforms associated to variants of the Laplace-Beltrami operators on the unit ball in $\mathbb{R}^{n}$, Indiana Univ. Math. J. 58(3) (2009), 1457-1492.

[18] Ungar, A. A., Thomas precession and the parametrization of the Lorentz transformation group, Found. Phys. Lett. 1 (1988), 57-89.

[19] Ungar, A. A., Thomas precession and its associated grouplike structure, Am. J. Phys. 59 (1991), 824-834.

[20] Ungar, A. A., Extension of the unit disk gyrogroup into the unit ball of any real inner product space, J. Math. Anal. Appl. 202(3) (1996), 1040-1057.

[21] Ungar, A. A., Thomas precession: its underlying gyrogroup axioms and their use in hyperbolic geometry and relativistic physics, Found. Phys. 27(6) (1997), 881-951.

[22] Ungar, A. A., Möbius transformations of the ball, Ahlfors' rotation and gyrovector spaces, In Themistocles M. Rassias (ed.): Nonlinear analysis in geometry and topology, Hadronic Press, Palm Harbor, FL (2000), $241-287$.

[23] Ungar, A. A., Analytic Hyperbolic Geometry - Mathematical Foundations and Applications, World Scientific, 2005.

[24] Ungar, A. A., Analytic Hyperbolic Geometry and Albert Einstein's Special Theory of Relativity, World Scientific, 2008.

[25] Ungar, A. A., From Möbius to gyrogroups, Amer. Math. Montly, 115(2) (2008), 138-144.

[26] Ungar, A. A., Barycentric calculus in Euclidean and hyperbolic geometry: A comparative introduction, World Scientific Publishing Co. Pte. Ltd., Hackensack, NJ, 2010.

[27] Ungar, A. A., Hyperbolic triangle centers: The special relativistic approach, Springer-Verlag, New York, 2010.

[28] Waterman, P.: Möbius transformations in several dimensions, Adv. in Math. 101 (1993), 87-113.

[29] Vahlen, K.: Über Bewegungen und komplexe Zahlen, Math. Annalen 55 (1902), 585-593. 